Cahiers
de la Recherche
surles
$D$ roits Fondamentaux

\section{Cahiers de la recherche sur les droits} fondamentaux

15 | 2017

Le corps humain saisi par le droit : entre liberté et propriété

\title{
Éthique biomédicale et droit de propriété sur le corps humain
}

Biomedical Ethics and Ownership Rights of the Human Body

\section{Bertrand Lemennicier}

\section{OpenEdition}

\section{Journals}

Édition électronique

URL : https://journals.openedition.org/crdf/544

DOI : $10.4000 /$ crdf.544

ISSN : 2264-1246

Éditeur

Presses universitaires de Caen

Édition imprimée

Date de publication : 1 novembre 2017

Pagination : 27-44

ISBN : 978-2-84133-858-0

ISSN : 1634-8842

\section{Référence électronique}

Bertrand Lemennicier, « Éthique biomédicale et droit de propriété sur le corps humain », Cahiers de la recherche sur les droits fondamentaux [En ligne], 15 | 2017, mis en ligne le 01 octobre 2019, consulté le 14 novembre 2022. URL : http://journals.openedition.org/crdf/544 ; DOI : https://doi.org/10.4000/crdf 544 


\title{
Éthique biomédicale et droit de propriété sur le corps humain
}

\author{
Bertrand LEMENNICIER \\ Professeur émérite à l'université Paris 2 - Panthéon-Assas \\ Centre de recherches en économie et droit (CRED)
}

I. Les lois sur la bioéthique sont fondamentalement nuisibles à la société

II. Une doctrine juridiquement mal fondée

A. La dichotomie entre la personne et la personnalité, entre le sujet et l'objet

B. Le corps humain comme fin ou comme moyen?

C. Conflit sur l'usage du corps humain, droit du premier occupant et droit de propriété sur soi

D. Cohérence du droit, inaliénabilité du libre arbitre et possession naturelle de soi

III. Une doctrine qui confond droit et morale

A. Droit positif ou droit moraliste?

B. La variété des approches morales

La répugnance de nos concitoyens à une liberté du commerce des organes destinés à la transplantation est notoire. C'est une anomalie. D'un côté, les gens reconnaissent qu'une augmentation de l'offre d'organes destinés à la transplantation sauve des vies humaines, et, de l'autre, ils s'opposent à l'achat et à la vente des organes comme moyen légal d'atteindre cet objectif.

Cette réticence prend sa source dans une proposition qui semble évidente en soi pour beaucoup de
Nous semblons être à la veille d'avoir la main sur le développement de notre corps, et même de notre cerveau. Avec la découverte des gènes, il semble que nous serions bientôt en mesure de contrôler le mécanisme de l'hérédité biologique.

Pierre Theillard de Chardin (1881-1955)

nos concitoyens. Les individus ne sont pas des objets commercialisables ni en pièces détachées ni en totalité. Le transfert d'un organe destiné à la transplantation d'un être humain à un autre contre de l'argent serait une atteinte à sa dignité humaine et à son intégrité. Cependant, le concept de dignité humaine n'est pas une proposition première indémontrable. Il n'est pas un axiome. Il repose lui-même sur une notion préalable: celle de personne humaine. Ce concept prend la forme concrète d'une

1. Cité par B. Jousset-Couturier, Le transhumanisme, Paris, Eyrolles, 2016, p. 99. 
réglementation des usages du corps humain qui s'imposerait à tous.

Par exemple, en quelques articles du Code de la santé publique (loi no 2011-814 du 7 juillet 2011 relative à la bioéthique), le législateur scelle la destinée d'un grand nombre de patients en attente d'un greffon:

Art. L. 665-10. La cession et l'utilisation des éléments et produits du corps humain sont régies par les dispositions du chapitre II du titre I $^{\text {er }}$ du livre I $^{\text {er }}$ du code civil et par les dispositions du présent titre.

Art. L. 665-11. Le prélèvement d'éléments du corps humain et la collecte de ses produits ne peuvent être pratiqués sans le consentement préalable du donneur. Ce consentement est révocable à tout moment.

Art. L. 665-12. Est interdite la publicité en faveur d'un don d'éléments ou de produits du corps humain au profit d'une personne déterminée ou au profit d'un établissement ou organisme déterminé. Cette interdiction ne fait pas obstacle à l'information du public en faveur du don d'éléments et produits du corps humain.

Cette information est réalisée sous la responsabilité du ministre chargé de la santé.

Art. L. 665-13. Aucun paiement, quelle qu'en soit la forme, ne peut être alloué à celui qui se prête au prélèvement d'éléments de son corps ou à la collecte de ses produits. Le cas échéant, les frais engagés peuvent être remboursés selon des modalités fixées par décret en Conseil d’État.

Art. L. 665-14. Le donneur ne peut connaître l'identité du receveur, ni le receveur celle du donneur. Aucune information permettant d'identifier, à la fois celui qui a fait don d'un élément ou d'un produit de son corps et celui qui l'a reçu, ne peut être divulguée.

Il ne peut être dérogé à ce principe d'anonymat qu'en cas de nécessité thérapeutique.

Ces articles sont à l'origine de la pénurie d'organes destinés à la transplantation et chaque année ils sacrifient, sur l'autel de la morale, la vie des patients dans la file d'attente $^{2}$. Il faut donc de sérieux arguments pour justifier de sacrifier la vie de tierces personnes pour satisfaire une conception particulière de la personne ou bien une morale téléologique du don pour le don dont les conséquences sont désastreuses pour certains patients.

Les usages de notre corps humain, consubstantiel à notre esprit et dont nous avons pour l'instant ${ }^{3}$ la possession naturelle, soulèvent une question simple: qui a le droit de dire ce que chacun peut ou doit faire de son corps: le Comité consultatif national d'éthique ${ }^{4}$, le législateur issu d'une majorité de rencontre, expression des préférences d'une faction politique «mandatée» par sa clientèle électorale, les croyances de nos voisins qui nous ostracisent si on ne partage pas leur vision du monde, notre conjoint qui manifeste souvent le désir de contrôler notre corps et/ou notre portefeuille, ou bien nous-même?

Il s'agit là d'un conflit traditionnel à propos des usages d'une ressource qui produit des services hautement demandés et sur laquelle les droits de propriété ne sont pas définis. L'économiste est en terrain familier, mais le juriste devrait l'être aussi puisqu'il s'agit de définir la notion de droit de propriété sur une ressource. Notre corps peut-il être en libre accès pour tous comme dans une pâture commune? Peut-il être géré sous forme d'anticommun, en collaboration entre plusieurs personnes détenant des droits d'exclusivité sur certaines parties de la ressource, ou plus simplement sous forme d'une propriété individuelle? La réponse de l'économiste est plutôt en faveur de la propriété individuelle, seule apte à allouer les usages de la ressource de manière dite « optimale», c'està-dire de telle sorte que tout autre usage ne rapporte pas une satisfaction supérieure. Mais cette solution est rejetée par le législateur, quitte à sacrifier la vie des patients en attente d'un greffon.

Nous allons tenter d'expliquer pourquoi le juriste fait fausse route lorsqu'il adopte, comme moyen de protéger l'individu, des droits de la personnalité fondés sur une théologie, au lieu d'adopter le concept de droit de propriété absolu de l'individu sur lui-même ou de libre disposition de son corps opposable à tout autre individu qui cherche à se l'approprier, pris isolément ou en groupe. Les débats sur le concept de personne ont un rôle crucial dans l'émergence des lois sur la bioéthique dont la nocivité

2. 579 patients demandeurs d'une transplantation sont décédés en 2015 dans la file d'attente, faute d'une offre suffisante.

3. Nous disons «pour l'instant» car la synergie entre les progrès scientifiques dans les nanotechnologies, la biologie, l'informatique, l'intelligence artificielle, les sciences cognitives et la robotique peut dans un avenir proche modifier «la nature» de l'homme et faire faire un saut dans l'évolution du corps humain.

4. Le fait même qu'il existe un Comité consultatif national d'éthique en dit long sur cette confusion du droit et de la morale. Cet aréopage a pour mission de donner des avis sur les problèmes éthiques et les questions de société soulevés par les progrès de la connaissance dans les domaines de la biologie, de la médecine et de la santé (voir art. L. 1412-1, modifié par la loi no 2004-80o du 6 août 2004, art. 1 $1^{\text {er }}$. En 2012, les personnalités désignées par le président de la République appartenaient aux différentes familles philosophiques et spirituelles: Ali Benmakhlouf (philosophe arabe), Michaël Azoulay (rabbin de la communauté juive de Neuilly-sur-Seine), Xavier Lacroix (professeur de philosophie et de théologie morale à l'université catholique de Lyon), André Glucksmann (normalien de l’École normale supérieure de Saint-Cloud, agrégé de philosophie, écrivain, aujourd'hui décédé) et Louis Schweitzer (pasteur des Églises baptistes, directeur de l'École pastorale de Massy). Le président actuel est Jean-Claude Ameisen (professeur d'immunologie à l'université Paris-Diderot/hôpital Bichat). Nous citons ces quelques noms nommés par le président de la République, mais ils sont au total trente-neuf membres nommés pour quatre ans et un président renouvelable tous les deux ans. Ce comité dispose d'un pouvoir d'influence en donnant des avis après saisines et autosaisines. Les rapports sont élaborés par des sous-comités composés de quelques membres. Par définition, les rédacteurs de ces rapports jouent un rôle essentiel en présentant le point de vue des personnes auditionnées d'une manière favorable à la vision des membres du comité. Le choix du président de la République de représentants des quatre religions ayant une audience sur notre territoire métropolitain ou de tel philosophe, laïc de service, est vraiment du domaine du prince. Les avis du comité n’ont strictement aucune valeur intrinsèque et aucun ne remet en cause le droit de la personne bien que le comité ait une pleine connaissance de ses effets pervers, comme le prouve l'avis 115 d'avril 2011 sur les questions relatives aux transplantations d'organes. 
pour les patients devient de plus en plus évidente au fur et à mesure que la pression de la demande augmente.

Dans ce qui suit nous développerons six idées:

- les lois sur la bioéthique sont fondamentalement nuisibles;

- la dichotomie entre la personne et la personnalité, entre le sujet et l'objet est au cœur de la doctrine juridique et il s'agit de faux concepts car les services rendus par les êtres humains et les choses sont substituables;

- en adoptant une définition du corps humain comme fin ou comme moyen, nous pouvons faire converger économistes, philosophes et juristes sur le concept de droit de propriété sur soi. Le problème des conflits entre les personnes à propos de l'usage du corps humain et de sa possession naturelle par celui qui y est incorporé, et / ou de celui des autres, soulève une question à laquelle il faut répondre: qui est le propriétaire de son corps, l'individu lui-même ou les autres pris individuellement (esclavage privé) ou en groupe (esclavage public)?;

- la solution proposée par l'économiste rejoint celle du juriste quand il doit définir à qui appartient une chose ou un objet que l'on vient de découvrir. C'est le droit du premier occupant ou du premier en possession;

- pour rendre compatibles ces droits de propriété entre eux, l'esclavage volontaire et le crime commandité sont bannis des relations contractuelles ou du droit des obligations;

- enfin, nous soulignerons combien les lois sur la bioéthique sont issues d'une doctrine qui confond le droit avec la morale. Elles imposent une règle de conduite dans les affaires concernant les usages du corps humain: celle du don pour le don au mépris de ses conséquences sur les autres individus et de règles de déontologie liées au respect du droit de propriété sur soi, seules règles permettant que restent compatibles entre elles les diverses règles morales adoptées par chacun d'entre nous.

\section{Les lois sur la bioéthique sont fondamentalement nuisibles à la société}

La répugnance de la société occidentale à traiter le corps humain comme un objet appropriable est immergée dans la religion chrétienne. Pendant quelques siècles la chrétienté a banni le taux d'intérêt comme le font toujours les musulmans. Elle a développé dans l'esprit des gens une réprobation pour tout ce qui touche au corps humain (dont les activités sexuelles). Elle a entravé le progrès médical en empêchant la dissection. Elle a fustigé la richesse et les inégalités. Cet atavisme perdure toujours dans nos sociétés contemporaines.

Par exemple, Michaël J. Sandel ${ }^{5}$ se plaint d'une société où tout est à vendre ${ }^{6}$ : les permis de polluer, la gestation pour autrui, le droit d'avoir une carte verte pour être un résident permanent aux États-Unis, un tag publicitaire sur votre peau, le pari sur la mort de quelqu'un avec un viager ou une assurance vie dont on paie les primes, les essais thérapeutiques d'une entreprise pharmaceutique sur votre corps, la queue dans une file d'attente à la place d'une autre personne contre rémunération, etc. En fait, il souligne un point important, qui nous intéresse pour le cas des transplantations d'organes, qui est la destruction par le marché des valeurs non marchandes importantes. Si vous payez des mercenaires pour faire la guerre à votre place, nous dit Sandel, cela épargne votre vie et celles de vos concitoyens mais détruit les valeurs liées à la citoyenneté7. Dans la même veine, légaliser le marché des organes détruit les valeurs liées à la morale du don pour le don, fondement philosophique des lois sur la bioéthique.

S'il en est ainsi, c'est sans doute qu'il y a des raisons. Le marché a une propriété essentielle: personne n'est contraint d'y participer. Le consentement des parties prenantes est le fondement de l'échange. Or, si tous les exemples mentionnés par Sandel révèlent la marchandisation d'un nombre croissant d'activités humaines, c'est que les individus en tirent un avantage mutuel, sinon ils n'agiraient pas ainsi.

Le refus de laisser les individus disposer de leur propre corps comme ils l'entendent - louer leur ventre, vendre leurs organes, cloner leur corps pour donner naissance à un être humain identique (un jumeau) ou manipuler leurs gènes pour choisir les caractéristiques corporelles de leurs propres enfants, procréer artificiellement, congeler les embryons pour une naissance dans deux siècles - est socialement nuisible. En effet, en refusant cela, on sacrifie toujours des vies humaines et on met en péril l'espèce elle-même. Aussi brutal que cela puisse paraître, si pour vivre un peu mieux une personne astreinte à une dialyse hebdomadaire recherche désespérément un rein naturel pour une transplantation et que d'autres personnes sont prêtes, moyennant rémunération de leur vivant ou après leur mort, à renoncer à un rein, un échange mutuellement bénéfique est possible.

5. M. J. Sandel, What Money Can't Buy: The Moral Limits of Markets, Londres, Penguin, 2012.

6. Nous critiquons les arguments avancés par cet auteur dans un autre texte: B. Lemennicier, Pénurie des organes à la transplantation: bioéthique et théorie économique, 2017, en ligne: http://lemennicier.bwm-mediasoft.com/displayArticle.php?articleId=783.

7. Sandel, à qui nous reprenons cet exemple, ne nous précise pas pourquoi la citoyenneté serait un bien. Elle peut l'être pour certains mais pas pour d'autres. C'est peut-être une bonne chose de voir disparaître cette notion de citoyenneté au profit du respect des droits de propriété des autres sur eux-mêmes. L'alternative au mercenariat est la conscription, c'est-à-dire le sacrifice forcé de toute une génération pour défendre par les armes un pouvoir politique que l'on peut désapprouver. La fraction des jeunes de la génération des années 1956-1962 enrôlés de force dans l'armée française et qui ont combattu en Algérie, considérée comme un territoire français à l'égal de la Corse d'aujourd'hui, pour une cause «injuste» - empêcher l'indépendance des Algériens, nationalistes, islamistes ou communistes, et qui ont pris fait et cause pour eux au péril de leur vie - ne devaient pas avoir la même conception de la citoyenneté que les gouvernants de l'époque. 
Empêcher un tel échange, c'est sacrifier la vie de la personne astreinte à la dialyse, en lui refusant une possibilité d'améliorer son bien-être. C'est aussi empêcher celui qui renonce à son rein, un pauvre par exemple, de gagner sa vie en mettant en valeur la seule ressource dont il dispose : les éléments de son corps. En imposant le don et la gratuité, le législateur fait reposer la transplantation d'organes sur les seuls donateurs altruistes dont il sait qu'ils ne sont pas suffisamment nombreux. Par ailleurs, il incite chaque personne sous dialyse à se porter demandeur d'une transplantation. Il crée une pénurie d'organes destinés à la transplantation. Une des conséquences de cette pénurie est la mort statistique de vies humaines.

Empêcher une femme stérile d'avoir un enfant grâce à une mère porteuse, c'est priver celle-ci des joies d'avoir son propre enfant et la condamner à se reporter sur le marché de l'adoption. C'est en même temps refuser à une autre femme le droit de gagner de l'argent comme elle l'entend. Empêcher la manipulation des gènes revient à priver nos enfants de l'opportunité de vies meilleures que les nôtres en leur évitant des maladies, en retardant le vieillissement et en allongeant la durée de vie ou encore en améliorant les performances de leur corps humain. C'est aussi priver les générations futures d'une plus grande diversité génétique. C'est aussi interdire à des communautés en voie d'extinction, les Pygmées, les Indiens d'Amazonie, les blancs d'Europe ou d'Amérique du Nord d'user d'un moyen qui leur éviterait de disparaître. La richesse génétique des Pygmées, des Indiens ou des blancs contribue tout autant que celle des noirs, des rouges ou des jaunes à la richesse de l'espèce humaine. La conservation et l'échange libre du matériel génétique des êtres humains est un moyen de préserver l'identité d'un groupe humain et sa survie autrement que par des règles de mariage draconiennes ou par des moyens usant de la violence politique comme des subventions aux familles nombreuses et l'exclusion des étrangers.

La nature produit des êtres humains qui naissent parfois avec deux têtes. D'autres naissent nains. Faut-il interdire la manipulation des gènes dans la crainte de voir les familles fabriquer des monstres dont ils tireraient profit en les exposant dans les foires? N'oublions pas une chose, très naturellement en se mariant on pratique la sélection des gènes. Faut-il interdire à des nains de se reproduire ensemble sous prétexte de voir naître un enfant nain qui n'a pas demandé à naître avec un tel handicap? L'homme et la femme atteints de nanisme ont procréé volontairement un enfant. Ils savaient que celui-ci ne mesurerait pas $1,80 \mathrm{~m}$.

C'est très exactement ce que nous proposent ceux qui veulent interdire les manipulations génétiques parce que l'enfant qui naîtrait n'aurait pas fait prévaloir son intérêt. Pourquoi exiger de ceux qui veulent des enfants dont les caractéristiques biologiques correspondent à leurs goûts ce que l'on n'exige pas de parents naturels?
Imaginons, comme dans le film Hibernatus, la naissance, aujourd'hui, d'un double de votre arrière-grand-père, dont l'embryon avait été congelé. Du coup, vos enfants à naître auront le même âge que votre arrière-grand-père. Imaginez les problèmes de filiation. Mais les problèmes de filiation n'existent qu'en vertu du droit à l'héritage. Vos enfants vivront comme frères et/ou sœurs avec un arrière-grand-père par la filiation qui aura les mêmes droits. Le problème de filiation n'existe pas en tant que tel. Les enfants n'ont aucun droit à hériter de leurs parents. C'est un privilège régalien qu'ils ont. En quoi les enfants auraient-ils un droit sur le fruit du travail de leurs parents? Les parents peuvent léguer leur fortune à leurs enfants ou à quelqu'un d'autre qu'ils apprécient mieux.

Revenons aux craintes de la fabrication en série de monstres ou de doubles de soi. L'eugénisme n'est pas une mauvaise chose en soi. On pratique l'eugénisme sur les plantes et les animaux. On crée de nouvelles plantes sans qu'il y ait de problèmes particuliers. Le diagnostic prénatal ou pré-implantatoire avec la procréation médicalement assistée (PMA) pour déceler des tares génétiques pouvant conduire à un avortement ou à une sélection des embryons dans une fécondation in vitro sont dans la ligne droite de l'eugénisme du XIX ${ }^{\mathrm{e}}$ siècle.

Interdire aux scientifiques de modifier la nature humaine, c'est priver les hommes des moyens de préserver leur vie face à des environnements hostiles sur notre planète ou en prévision de voyages sur d'autres planètes. La génétique ouvre des horizons pour améliorer la résistance des êtres humains à des environnements très hostiles à la nature humaine. Ainsi, beaucoup d'espèces très primaires sur Terre supportent déjà des températures très basses ou très fortes et résistent aussi au rayonnement solaire; leur génétique peut ouvrir des pistes pour renforcer les protections «naturelles» de l'homme dans ces environnements où sa constitution actuelle l'empêche de survivre. Toutes ces recherches soulèvent des craintes. L'apparition d'une race de «surhomme», pensée et créée par l'homme, heurte l'esprit de nos contemporains. Pourtant, la nature l'a déjà fait, à l'image de l'Homo sapiens surclassant l'homme de Néandertal grâce au FOXP2 qui facilite la communication par la parole et l'apprentissage. Et alors? Croyez-vous que l'espèce humaine ne mérite pas d'être améliorée? Le transhumanisme suscite des vocations. Chez Google X Lab, des dépenses de recherche particulièrement importantes ont été consacrées à ce défi. En fait, l'Homo sapiens 2.0 pourrait bien devenir "la première espèce "libre" au sens où il échapperait aux incertitudes de la sélection naturelle ${ }^{8}$.

Grâce aux nanotechnologies, biotechnologies, informatique et sciences cognitives (NBIC), la possibilité de changer l'évolution de la nature de l'homme par des mutations génétiques contrôlées et non par le hasard de la sélection naturelle est à portée de main. Cette opportunité est une chance pour l'espèce humaine qui pourrait s'acclimater à 
des environnements hostiles à notre constitution biologique sur Terre ou dans la conquête de l'espace. La liberté de disposer de son corps et d'en exploiter toutes les ressources offre aux individus la possibilité d'augmenter la résistance de leur corps face aux maladies et au vieillissement, mais aussi d'améliorer leurs performances dans l'exercice physique et intellectuel.

Ne vous méprenez pas sur le type d'améliorations que le marché fera apparaître. Imaginez une nouvelle race d'hommes-oiseaux créée par manipulation génétique. Un rêve qui remonte à la mythologie. Ils ont des ailes et tels des anges volent. Aujourd'hui, les hommes volent déjà. Ils volent avec des avions, des ultra-légers motorisés (ULM), des deltaplanes. Mais, naturellement, il est plus commode de voler avec un avion, un ULM ou un deltaplane qu'avec des ailes issues d'une manipulation génétique. La raison en est simple. On laisse son ULM au garage, en revanche, si nous avons des ailes, comme un oiseau, par la force des choses, il faudra les garder avec soi et, compte tenu du poids de notre corps, nos ailes auront la taille d'un deltaplane. Imaginez les conséquences: être obligé de vivre au sommet des gratte-ciel ou dans des montagnes près d'endroits qui tombent à pic pour nous lancer dans les airs. Non, nous ne le ferons pas. Mais si, pour des raisons militaires, l'État a besoin d'hommes-oiseaux, il n'hésitera pas à les créer. Il faut donc de solides raisons pour interdire à chacun d'entre nous de disposer librement de notre corps humain et imposer cette interdiction à l'ensemble de la collectivité.

\section{Une doctrine juridiquement mal fondée}

Le juriste positiviste est paradoxalement très influencé par la vision de la théologie chrétienne où le corps humain est consubstantiel à la personne. La réflexion juridique, écrit Jean-Michel Poughon,

[...] semble prolonger la théologie en en reprenant la logique d'un fondement transcendantal à la dignité humaine. D'autre part, elle tend à ériger ce fondement en dogmatique. Cette approche apparaît clairement en ce qui concerne la propriété du corps. Consubstantiel à la personne, il est donc indisponible ${ }^{9}$.

Nous avons déjà critiqué le concept de droit de la personne dans un article ancien, datant de $1991^{10}$. Vingt-cinq ans après sa rédaction, le diagnostic nous paraît toujours identique. Le droit de la personne, inscrit dans le droit positiviste contemporain, est philosophiquement mal fondé. Dans le texte qui suit, il nous paraît important de réfléchir à la définition de la personne.

La dichotomie entre le corps humain (objet) et la personne (sujet) se heurte à l'incorporation réciproque de la personne dans une machine biologique, auto-reproductible et biodégradable appelée «corps humain». On doit à Descartes ${ }^{11}$ cette idée de comparer le corps humain à une machine support de la pensée. Elle a permis de jeter un regard différent sur l'être humain et a ouvert la voie au développement scientifique de la médecine.

Les médecins agissent, dans leur pratique, comme si le corps était un objet. Mais simultanément, ils adhèrent à la théologie chrétienne de la consubstantialité du corps (objet) et de la personne (sujet). Ce qui crée, chez eux, une dissonance cognitive. De même, les associations de défense des patients en attente d'un greffon soutiennent les lois sur la bioéthique. Elles en connaissent les effets pervers par le décès prématuré de certains de leurs membres. Or, elles sont censées défendre les patients mais elles refusent un marché libre des organes destinés à la transplantation qui pourraient les sauver. Là aussi nous observons un paradoxe puisque les victimes de cette législation adhèrent à la conception théologique de la personne qui les tue ${ }^{12}$. Les personnes à la tête de ces associations devraient se poser des questions sur les jugements de valeur qui guident leurs actions, surtout quand leurs conséquences affectent la vie d'autres personnes qui ne partagent pas nécessairement leur doctrine philosophique.

La conception de l'individu ou de la personne mérite, en fait, un dialogue entre médecins, juristes, philosophes et économistes puisque seuls les économistes soutiennent, en théorie, un marché libre des organes destinés à la transplantation. Un marché libre se définit par trois attributs : des droits de propriété bien définis, le consentement à l'échange de ces droits et la non-interférence dans le système de prix par des moyens usant de la coercition privée ou publique.

L'économiste n'est pas uniquement le spécialiste de la science des choix (praxéologie) même s'il apparaît souvent comme tel à nos contemporains lorsque ces derniers parcourent les revues académiques. L'économie est surtout une science des contrats. Le Prix Nobel ${ }^{13}$ James M. Buchanan l'a bien compris. Il écrit en 1975 :

L'économie est plus proche de la science des contrats que de la science des choix. Le principe de maximisation doit être remplacé par celui de l'arbitre qui s'efforce de résoudre des conflits entre individus [...] avec pour principe unificateur les gains de l'échange ${ }^{14}$.

9. J.-M. Poughon, «L'individu propriétaire de son corps? Une réponse entre scolastique juridique et réalisme économique?», L'Europe des libertés, $\mathrm{n}^{\circ} 11,2003$, en ligne: $\mathrm{http}$ ://leuropedeslibertes.u-strasbg.fr/article.php?id_article=100\&id_rubrique $=5$.

10. B. Lemennicier, "Le corps humain: propriété de l’État ou propriété de soi ?», Droits, nº 13, 1991. Voir aussi B. Lemennicier, La morale face à l'économie, Paris, Éditions d'Organisation, 2005, chap. II.

11. R. Descartes, Les principes de la philosophie [1644], Paris, Arvensa Éditions, 2015, première partie, titre VIII.

12. En 2015, 579 personnes en attente d'un greffon sont décédées, soit 37 décès pour 1000 patients. La mortalité sur les routes en 2013 était de 5,8 tués pour 100 ooo habitants ou encore 0,058 tué pour 1000 habitants, en supposant que tous les habitants soient des automobilistes.

13. Ce que nous désignons par Prix Nobel d'économie est plus justement le prix de la Banque de Suède en sciences économiques en mémoire d'Alfred Nobel.

14. J. M. Buchanan, «A Contractarian Paradigm for Applying Economic Theory», American Economic Review, vol. 65, nº 2, 1975, p. 229 (nous traduisons). 
La science des choix n'est pas fondamentale à l'économiste, elle est un instrument d'analyse pour comprendre le comportement des individus. En revanche, la science des contrats ou de l'échange l'est. Celle-ci a pour objet la coordination des plans des individus entre eux pour obtenir une constellation sociale, politique et économique où tous les individus, dans une période donnée, réalisent leurs anticipations. Et si tout le monde réalise ses anticipations, les conflits interindividuels ont disparu. C'est la paix civile. Et c'est là où le juriste et l'économiste se rejoignent et vont s'affronter sur les moyens utilisés pour atteindre ce «nirvana» car le droit a pour objet de «prévenir ou régler les conflits qui naissent dans la vie de la société $»^{15}$.

Le droit de propriété et son transfert volontaire dans d'autres mains sont donc considérés par l'économiste comme le moyen de pacifier les relations entre les individus ou personnes à propos des services rendus par les objets ou les personnes. Par conséquent, le droit de propriété de la personne sur elle-même devrait être le moyen de minimiser les conflits qui surgissent entre les individus à propos des services rendus par les choses ou les personnes.

Or, tel n'est pas le cas. Le droit de propriété sur soi, c'est-à-dire sur les usages que chacun peut faire de son corps humain, est nié par le droit de la personne ou les droits de la personnalité. Les économistes spécialistes de la théorie des choix publics sont accoutumés à toutes ces réglementations issues des groupes de pression qui cherchent à influencer le législateur au détriment de la fonction naturelle du droit qui consiste à pacifier les relations interpersonnelles au lieu de les accroître. De fait, les droits de la personne ou de la personnalité sont des réglementations sur l'usage du corps humain qui augmentent les conflits au lieu de les réduire. Les droits de la personne ne sont pas du droit tel que le juriste et l'économiste l'appréhendent dans sa finalité. La doctrine des droits de la personne repose fondamentalement sur la dichotomie sujet-objet, mais que vaut cette dichotomie? Abordons ce point.

\section{A. La dichotomie entre la personne et la personnalité, entre le sujet et l'objet}

Les êtres humains seraient dotés de certaines capacités que l'on ne retrouve pas chez les autres êtres vivants, en particulier les animaux. Mais rien n'est moins sûr. Les animaux vivent une existence séparée des autres animaux de leur espèce. Ils sont aussi dotés de certaines capacités de langage, de réflexes préprogrammés, de sentiments ${ }^{16}$, d'instincts de reproduction entre mâle et femelle ou encore respectent des règles de conduite pour pacifier les conflits qui naissent entre eux à propos de la propriété territoriale (les chats) ou des règles de soumission au chef de la meute pour s'approprier la nourriture et s'accoupler avec des femelles (les loups). On retrouve aussi toutes ces caractéristiques chez l'être humain. Qu'est-ce qui peut bien différencier l'homme de l'animal ${ }^{17}$ ? L'homme possèderait-il tous les attributs des animaux à un plus haut degré? Aristote a une réponse simple:

L'homme, seul de tous les animaux, possède la parole. [...] Le discours sert à exprimer l'utile et le nuisible, et, par suite aussi, le juste et l'injuste car c'est le caractère propre de l'homme par rapport aux autres animaux, d'être le seul à avoir le sentiment du bien et du mal, du juste et de l'injuste et des autres notions morales et c'est la communauté de ces sentiments qui engendre famille et cité ${ }^{18}$.

Chez Aristote, les notions de bien ou de mal n'ont aucun sens en dehors de la vie sociale puisqu'elles émergent des rapports entre l'être humain et ses semblables. Or, nous dit-il, le processus de sélection naturelle (ou la nature), «qui ne fait rien en vain ${ }^{19}$, nous a doté de la faculté spéciale de discerner le bien du mal et de la parole pour la communiquer. En conséquence, la capacité de distinguer le bien du mal, le juste de l'injuste, l'utile du nuisible, offre l'occasion de développer des règles de conduite et la "rectitude» avec laquelle on les respecte. Ce corps de règles définirait le droit ${ }^{20}$.

Reportons-nous à une autre définition, plus récente cette fois: «La personnalité c'est l'individu, soit l'ensemble des aspects d'une personne qui la distingue de tous ses semblables passés, présents et futurs ${ }^{21}$.

Bernard Beignier utilise cette façon de caractériser à la fois la personnalité et la personne. Dans cette définition, la personnalité est ce qui distingue une personne de toutes les autres personnes, le nom par exemple ou son identité ou bien son caractère, son courage, son intelligence, sa méchanceté, etc. Toutes ces caractéristiques sont finalement des attributs que les autres personnes perçoivent

15. G. Ripert, Les forces créatrices du droit, Paris, LGDJ, 1955, p. 71.

16. Leur nature leur permet d'exprimer des sensations de douleur et de joie et de les communiquer aux autres. Tous les détenteurs d'animaux de compagnie en ont l'expérience.

17. Certains philosophes et moralistes contemporains évoquent le «droit» des animaux en refusant de les traiter comme des objets ou des biens meubles. Auquel cas le droit ne serait plus relatif à l'espèce humaine. Après tout, il a fallu du temps pour que l'esclave cesse d'être traité comme un objet qui est bien le traitement que l'on accorde aux animaux «domestiques». Comme l'écrit le poète Hésiode, cité par Aristote qui le commente: « une maison en premier lieu, ainsi qu'une femme et un bœuf de labour» (Aristote, La politique, J. Tricot (trad.), Paris, J. Vrin, 1989, I, 2, p. 26), car le bœuf tient lieu d'esclave aux pauvres. On mesure mieux l'importance du langage et l'impossibilité de communiquer pour élaborer une pensée. Imaginons deux minutes que votre chat monte sur la table au moment de dîner et commence à discuter avec vous par la parole au lieu de le faire avec sa patte tout en ronronnant ou en miaulant à fendre l'âme, comment allez-vous le considérer, toujours comme un animal?

18. Aristote, La politique, I, 2, p. 29.

19. Ibid.

20. Voir M. Villey, Questions de Saint Thomas sur le droit et la politique ou le bon usage des dialogues, Paris, PUF, 1987, p. 114-115.

21. B. Beignier, Le droit de la personnalité, Paris, PUF (Que sais-je?), 1992, p. 6. 
subjectivement dans le comportement de cet individu. Cela ne définit pas cependant la personne (physique) ${ }^{22}$. De son côté, le philosophe Stéphane Chauvier écrit:

Le mot "personne» [ou personnalité chez Beignier] semble en effet avoir deux sens différents selon que l'on dit d'un être [d'un individu] qu'il est une personne ou selon que l'on parle de la personne qu'il est.

Un peu plus loin il écrit:

Une personne est un être doté d'une aptitude à la conscience de soi. En ce sens un homme est une personne tandis qu'une pierre n'en est pas une. Mais lorsque nous parlons de la personne qu'il est [il s'agit de] désigner le contenu de sa conscience de soi $^{23}$.

Retenons pour l'instant cette façon de séparer la personnalité de la personne. Cela peut vouloir dire que le mot "personne» décrit tous les êtres capables de se penser soi-même, caractéristique commune à l'espèce humaine, alors que la personnalité décrit un individu singulier se pensant lui-même avec un projet de vie qui concourt à son épanouissement en tant qu'être humain. Or, ce n'est pas la personne en tant qu'attribut commun à une espèce qui intéresse le droit de la personnalité, mais plutôt la personnalité telle que souhaitée par l'individu lui-même. Tout devient subjectif et relatif, le droit disparaît en faveur du cas par cas, chacun voulant réaliser l'épanouissement de sa personnalité réclame une règle de droit qui facilite ce "human flourishing» ${ }^{24}$ ou dans les termes d'Aristote cette «eudaimonia».

Pour mesurer les impasses et la subjectivité de cette approche relativiste on peut prendre l'exemple du transsexualisme qui fait exception à l'intangibilité du corps humain. Le sexe est un attribut commun soit aux hommes soit aux femmes. Il est un sous-ensemble (le genre) de l'espèce : être humain. Une femme est-elle une personne ou une personnalité? Quand la Cour de cassation ne fait pas obstacle ${ }^{25}$ à la demande d'un homme de passer d'un sous-ensemble à l'autre, elle accepte la conception philosophique de la séparation entre la personne et la personnalité et la définition de la personnalité comme l'expression d'un être se pensant lui-même femme tout en étant «objectivement» un homme. Du même coup, elle contredit la philosophie des droits de la personnalité tels que définis par Bruno Petit et Sylvie Rouxel dans leur manuel sur le droit des personnes:
[...] l'ensemble des prérogatives, indissociables de l'individu, qui expriment la personnalité de celui-ci [...]. Attachés à l'individu [ces droits de la personnalité] trouvent leur source dans le seul fait de sa naissance, ils sont innés, ne s'acquièrent pas et ne résultent pas de l'activité de la personne ${ }^{26}$.

Si certains traits de la personnalité sont innés, être de sexe masculin ou féminin, intelligent ou non, violent ou non ${ }^{27}$, tous ne le sont pas. Ils s'acquièrent et résultent souvent de l'activité de la personne, de la formation de ses croyances par son environnement social. Ils sont évolutifs. On ne sait jamais s'il s'agit d'un rôle public ou d'un moi profond. Est-ce qu'un comédien a une personnalité? Non, il en a plusieurs, celles de tous les personnages qu'il va jouer. Des personnalités issues de la conscience de soi d'un auteur qui représente peut-être la personnalité qu'il aurait aimé être, mais qui n'est pas sa personnalité.

Prenons le fœetus ou un nouveau-né, un jeune enfant ou encore un individu dans un coma dépassé, un schizophrène, un trisomique 21, doit-on les inclure dans les personnes? Ceux qui n'ont pas la parole ne peuvent pas prouver qu'ils ont une quelconque personnalité. Celui qui a des troubles de la personnalité est-il une personne? La réponse est négative. Ils seront traités comme des animaux domestiques dont les prérogatives juridiques se traduisent par un droit de garde dont la fonction est de protéger cet être vivant et non de le martyriser, principe étendu aux animaux domestiques.

Maintenant prenons un robot, qui parle, raisonne, fait des choix, crie si on lui tape dessus ${ }^{28}$, s'auto-répare, gagne des compétitions d'échecs, nous dit qu'il se pense par lui-même et pétitionne pour sa liberté en quittant l'atelier de son créateur sans son consentement. Est-il une personne? Nous savons qu'il s'agit d'un non-existant, créé et fabriqué par une personne qui en a la propriété. Ce robot peut être vendu, démonté, recyclé, rafistolé, amélioré plus facilement qu'un animal ou un être humain. Est-ce que la personne qui en a la propriété peut invoquer la primauté de la personne pour «son" robot et interdire toute atteinte à la dignité de celui-ci, même si l'on sait pertinemment qu'il s'agit d'un objet? Pour le créateur de robot, le robot est une personne avec une personnalité, or c'est un non-existant, un objet comme une pierre peut l'être. Il n'a pas conscience de lui-même, mais comment peut-on le savoir puisqu'il communique par la parole

22. La seule qui nous intéresse ici par opposition à la personne morale où le juriste fait comme si les représentants d'un contrat entre plusieurs personnes avaient une personnalité propre.

23. S. Chauvier, Qu'est-ce qu'une personne?, Paris, J. Vrin, 2012, p. 11.

24. D. B. Rasmussen, D. J. Den Uyl, Liberty and Nature: An Aristotelian Defense of Liberal Order, La Salle, Open Court, 1991, p. 37.

25. Cass., Ass. plén., 11 décembre 1992, 2 arrêts, nº 91-1190o et 91-12373, Bulletin, n ${ }^{13}, 1992$; cité dans B. Petit, S. Rouxel, Droit des personnes, $4^{\mathrm{e}}$ éd., Grenoble, Presses universitaires de Grenoble, 2015, $n^{\circ} 97$, voir aussi $n^{\circ} 112$

26. B. Petit, S. Rouxel, Droit des personnes, $\mathrm{n}^{\circ}$ 22. Les deux auteurs parleront d'exception à la règle, mais il s'agit bien ici d'une contradiction au principe d'universalité de la règle de droit et à son applicabilité. Si je me pense moi-même comme un "gourou " qui s'épanouit en modifiant la personnalité des autres, pourquoi m'empêcher au nom de l'intangibilité de la personne d'exercer cette activité?

27. Le sexe et la violence sont en général des traits de caractère liés aux chromosomes humains si l'on prend la population carcérale comme un indicateur du lien génétique entre sexe, violence et chromosomes humains. Mais il y a toujours des exceptions comme avec le transsexualisme ou les violences infra-familiales.

28. Un humanoïde de type «HRP-2 Promet» produit par les Japonais ou un «émorobot» du programme Feelix Growing; mentionnés par B. JoussetCouturier, Le transhumanisme, p. 115. Les chercheurs élaborent aujourd'hui des robots pouvant interagir émotionnellement avec les humains. 
et prétend se penser lui-même puisque son créateur l'a programmé pour cela.

Comme cette discussion le suggère, nous considérons le corps humain comme un «objet» avec un sujet (ou un individu ayant développé une personnalité) incorporé en son sein. Cette incorporation de la personnalité dans une matière animée ou vivante nous rappelle immédiatement les droits de la personnalité des auteurs sur leurs œuvres artistiques, plus connus sous le nom de droit moral de l'auteur. Tout ou partie de la personnalité de l'auteur de l'œuvre est incorporé dans un objet inanimé (une feuille de papier, une bobine de film, un tableau, un spectacle, etc.) dont la particularité est d'être un non-existant.

Maître Sabine Haddad résume brièvement le droit moral de l'auteur tel qu'on le trouve défini dans le Code de la propriété intellectuelle par quatre caractéristiques principales:

L'auteur jouit du droit au respect de son nom, de sa qualité et de son œuvre. [...] [Ce droit] est perpétuel, inaliénable et imprescriptible. Il est transmissible à cause de mort aux héritiers de l'auteur.

[...]

Il est incessible, même par donation. L'auteur ne peut $\mathrm{y}$ renoncer. A fortiori toutes clauses portant renonciation, transfert ou cession du droit moral, est nulle [sic].

[C'est] un droit imprescriptible. Celui qui n'use pas de son droit durant un certain délai, ne le perdra pas. [...]

[C'est] un droit insaisissable. Sans l'accord de l'auteur, pas de commercialisation ou de saisie ${ }^{29}$.

En conséquence, l'acheteur d'une œuvre d'art ne peut pas l'altérer ni la détruire sans le consentement de l'auteur même si ce dernier ne respecte pas les préférences de l'acheteur exprimées lors d'une commande. Le droit fait alors d'un non-existant une personne. Qu'il y ait des servitudes sur l'objet n'est pas en soi un problème car il s'agit finalement d'un pari sur la valeur de l'objet en présence d'une telle clause au contrat de transfert. S'il y a consentement des deux parties, l'acheteur anticipe cette servitude et pense qu'elle améliorera la valeur de l'œuvre d'art. Sinon l'acheteur aurait renoncé à cette transaction. Mais que le juge ex post, face à une œuvre qui ne correspond pas à la commande, interdise la destruction de l'objet échangé au prétexte que la personnalité du créateur est incorporée dans cet objet, devenu de ce fait insaisissable, soulève une interrogation. Cette dernière caractéristique, qui présuppose une indissociabilité entre l'objet et la personne ${ }^{30}$ et donc une incorporation de la personne dans un objet, fonde le droit de la personnalité.

La consubstantialité de la pensée et du corps humain est mise à mal par le juriste lui-même avec le concept de droit moral de l'auteur. D'un côté, la doctrine juridique soutient la proposition suivante: si la personnalité (P) est incorporée dans le corps humain (C), un existant, la personnalité étant consubstantielle au corps humain, alors ce dernier (C) n'est pas un objet (O). De l'autre côté, avec le droit moral de l'auteur, la doctrine juridique soutient la proposition suivante: si la personnalité $(\mathrm{P})$ de l'individu est incorporée dans un objet $(\mathrm{O})$, un non-existant, alors l'objet $(\mathrm{O})$ est consubstantiel à la personnalité $(\mathrm{P})$. Cet objet $(\mathrm{O})$ acquiert les propriétés du corps humain $(\mathrm{C})$ ou est traité comme tel. Les deux propositions semblent contradictoires $^{31}$. En droit de la propriété intellectuelle, l'incorporation de la personnalité dans les objets donne à ces derniers un statut de corps humain avec des droits patrimoniaux et extrapatrimoniaux. En droit de la bioéthique, l'incorporation de la personnalité dans le corps humain interdit de traiter ce dernier comme un objet avec les droits patrimoniaux qui vont avec.

Cette vision philosophique de la personne ou de la personnalité ne nous dérangerait pas si elle n'était pas en contradiction avec les attributs que l'on exige habituellement d'un droit. Ce dernier doit être généralisable, non ambigu, facilement identifiable et clair dans son expression, non contradictoire avec d'autres droits, stable pour être parfaitement prévisible, non rétroactif pour ne pas tromper les attentes des individus, ne pas exiger l'impossible, être applicable ou sans écart entre la déclaration de la loi et son application par l'administration qui a la charge de la mettre en œuvre ${ }^{32}$. Rien de tout cela avec les droits de la personnalité. Ils sont ambigus (transsexuels), peu identifiables

29. S. Haddad, «Les cinq caractères du droit moral de l'auteur», Legavox, 2012, en ligne: http://www.legavox.fr/blog/maitre-haddad-sabine/cinqcaracteres-droit-moral-auteur-9811.htm\#.V9WKYiiLShc.

30. C'est ce que l'on appelle en théologie la consubstantialité de la personne et du corps humain. L'indisponibilité du corps humain de la loi sur la bioéthique de 1994 révèle bien le caractère religieux de son inspiration.

31. Appelons P le concept de personnalité, qui est une œuvre de l'esprit, une propriété incorporelle; C, celui du corps humain, un objet animé; O celui d'un objet inanimé appropriable sous forme d'une propriété matérielle; I, le concept d'incorporation. Le signe $\rightarrow$ signifie «implique»; le signe $\equiv$ signifie «identique à »; enfin, le signe indique «négation de». Nous avons donc dans le cas des lois sur la bioéthique la proposition suivante: $\mathrm{P}$ I C $\rightarrow \mathrm{C} \equiv \sim \mathrm{O}$. La personnalité $\mathrm{P}$, propriété incorporelle, incorporée dans $\mathrm{C}$, le corps humain, implique que $\mathrm{C}$ n'est pas identique à un objet, O. En conséquence, le corps humain n'est pas une propriété matérielle. Dans le cadre du Code de la propriété intellectuelle, la propriété incorporelle (par exemple création d'une personnalité) est définie par l'article L. 111-1 qui nous dit: l'auteur d'une œuvre de l'esprit jouit sur cette œuvre, du seul fait de sa création, d'un droit de propriété incorporelle exclusif et opposable à tous. L'article L. 111-3 précise quant à lui que cette propriété incorporelle est indépendante de la propriété de l'objet matériel dans lequel elle est incorporée. Nous avons donc la proposition suivante: $\mathrm{P}$ I O $\rightarrow \mathrm{O} \equiv \mathrm{C}$. Le concept de personnalité, une propriété incorporelle, une fois incorporé dans un objet (inanimé comme un tableau) implique que cet objet devienne identique au corps humain $\mathrm{C}$ et est donc de ce fait dans un état d'indisponibilité comme le corps humain. Ce qu'il est en partie. Les deux propositions, c'est-à-dire les deux lois, prises ensemble nient une loi fondamentale de la logique classique, celle de l'identité: une chose est une chose ou n'est pas une chose, $\mathrm{O} \equiv \mathrm{C}$ ou $\mathrm{C} \equiv \sim \mathrm{O}$. Le paradoxe s'accentue avec l'article L. 111-3 du Code de la propriété intellectuelle qui nous dit que la propriété incorporelle est indépendante de la propriété de l'objet matériel. Pourquoi le législateur n'en fait-il pas autant avec les lois sur la bioéthique en rendant la propriété sur sa personnalité indépendante de la propriété sur son corps humain, un objet animé mais matériel dont nous avons la pleine possession?

32. Voir L. L. Fuller, The Morality of Law, New Haven - Londres, Yale University Press, 1964, chap. II. 
(plusieurs personnalités peuvent coexister dans un même corps humain), en contradiction avec d'autres droits (droit moral de l'auteur), non généralisables (la personnalité varie en fonction des époques, de la localisation géographique, de l'âge de l'intéressé), difficilement prévisibles puisque ces droits sont le produit de majorités éphémères au Parlement.

Tournons-nous vers une autre définition de la personnalité, celle proposée par Alain Sériaux (1992):

Les personnes physiques sont les êtres humains concrets, singuliers. Les juristes ont toujours reconnu leur existence en les distinguant des biens ou des choses. Ces derniers sont essentiellement susceptibles d'appropriation: ils sont destinés à appartenir à des personnes. Les personnes physiques au contraire se caractérisent par le fait qu'il s'agit d'êtres libres, autonomes, qui ne peuvent devenir la chose d'autrui ${ }^{33}$.

L'auteur nous dit que les choses ou les objets sont appropriables à l'inverse des personnes physiques qui ne peuvent pas l'être. Mais il précise que celles-ci ne peuvent «devenir la chose d'autrui » ${ }^{34}$. Cette définition négative et impérative n'est pas dénuée d'intérêt. On ne cherche pas à définir positivement ce qu'est une personne mais négativement. Une personne est un individu qui n'est pas l'esclave d'un autre individu. Ce dernier ne peut pas user d'une personne comme il userait d'un objet dont il a la propriété. Sériaux s'inscrit dans une vision où le droit dicte ce que les individus peuvent faire dans leur relation interpersonnelle à propos des choses et des personnes. La propriété des choses, rappelons-le, est un panier de droits qui décrit ce que l'individu peut faire avec les ressources dont il a la possession. Si le corps humain est « juridiquement » une chose, les droits de propriété décrivent ce que le propriétaire peut en faire.

\section{Ainsi il peut:}

1. user de son corps comme il l'entend (consommation de drogues);

2. le transformer (changer de sexe, se mutiler - circoncision -, modifier la taille de ses seins au moyen de la chirurgie esthétique, etc.);

3. le détruire (suicide, euthanasie);

4. l'abandonner (grève de la faim, anorexie);

5. le transmettre en partie ou en totalité à autrui (donner ou vendre une partie de son corps - organes destinés à la transplantation, gènes, etc. -, ou la totalité du corps après décès);

6. transférer l'usage de son corps à autrui (servage, esclavage volontaire);

7. exclure autrui de l'usage de son corps (avortement);

8. le conserver à perpétuité (momification ou cryogénisation, clonage);
9. jouir des revenus tirés de cette ressource (louer ou vendre tout ou partie de son corps humain, son cerveau, sa voix, son ventre, son sexe, ses jambes).

En revanche, il ne peut en faire une utilisation:

10. qui endommage la propriété d'autrui (coups, blessures, homicides volontaires).

Et si quelqu'un endommage ou vole cette ressource, il est en droit d'exiger :

11. la restitution de la ressource, ou une compensation monétaire.

Les droits de la personne seraient donc un ensemble de restrictions aux items du panier de droits qui définit la propriété. Excepté les points 10 et 11 qui assurent normalement l'intégrité physique de chaque individu, tous les autres items sont «juridiquement» niés par le législateur. Par contrecoup, ce dernier s'arroge le droit de décider de l'usage du corps humain de chacun à la place des individus eux-mêmes. C'est lui qui dicte, au nom d'une clientèle électorale qui l'a porté au pouvoir, la façon dont on doit employer les ressources tirées de son corps. Le législateur traite alors chacun d'entre nous comme un esclave en affirmant un droit de propriété sur le corps humain de «ses sujets». Seuls les hommes d'État auraient le droit d'être propriétaires du corps des individus puisque l'esclavage privé est interdit. D'ailleurs, les rédacteurs de l'article 16 du Code civil qui, dans son chapitre II, définit le respect du corps humain, concluent: «Les dispositions du présent chapitre sont d'ordre public».

On ne peut pas être plus clair, le corps humain est à la merci de la conception que se fait le législateur de l'ordre public. En particulier, le législateur, sous la pression de la corporation des juristes, des médecins et des Églises (en particulier l'Église catholique), a décidé que les droits de la personnalité ou de la personne (la confusion entre les deux concepts est souvent entretenue) sont hors commerce. Ils ne sont pas évaluables en argent. Ils sont incessibles, insaisissables, absolus et reconnus à toute personne physique ou considérée comme telle. Cette attitude socialement nuisible ne diffère pas de toute autre législation dont les effets pervers sont désastreux. On observe un processus d'intervention croissante pour éliminer ces effets pervers au lieu de revenir sur la législation qui les a induits ${ }^{35}$.

\section{B. Le corps humain comme fin ou comme moyen?}

Un moyen de réconcilier les juristes, les philosophes et les économistes avec les faits réels ou les forces créatrices du droit serait de s'inspirer du point de vue de deux

33. A. Sériaux, Les personnes, Paris, PUF (Que sais-je?), 1992, p. 5.

34. Pendant très longtemps l'esclavage privé a été toléré dans les sociétés occidentales.

35. Le salaire minimum est un excellent exemple d'une telle législation. Il crée un chômage dit de file d'attente. Pour faire patienter les chômeurs qui espèrent être embauchés dans un emploi payé au-dessus du prix du marché, le législateur crée le revenu de solidarité active (RSA). Ce dernier, dont l'objet est de corriger l'effet pervers engendré par le salaire minimum, accroît la demande d'éligibilité à ce statut de "pauvre » et augmente la durée de cette situation de pauvreté au fur et à mesure où le niveau de cette aide se rapproche du salaire minimum. 
philosophes: Emmanuel Kant et Robert Nozick. Relisons ce qu'écrit Kant (1785):

Agis de telle sorte que tu traites l'humanité, soit dans ta propre personne soit dans celle des autres, toujours comme une fin et jamais comme un moyen.

Et d'ajouter :

Agis uniquement d'après la maxime qui fait que tu peux vouloir en même temps qu'elle devienne une loi universelle ${ }^{36}$.

Non seulement on ne peut traiter les autres personnes comme des moyens pour atteindre ses fins, seul ou en groupe (via l'État par exemple), mais on ne pourrait pas non plus utiliser sa propre personne comme moyen pour atteindre ses propres fins, par exemple consommer de la drogue pour écrire des romans ou consommer de l'érythropoïétine (EPO) pour être champion olympique du 100 mètres, ou encore vendre l'un de ses reins pour sortir de la pauvreté, se cloner ou modifier génétiquement sa descendance ou pratiquer l'eugénisme ${ }^{37}$. Il s'agit bien là de discuter de l'usage de son corps humain, vu comme moyen d'atteindre l'épanouissement de sa propre personne ou personnalité. Traiter les autres comme une fin et non comme un moyen pour atteindre ses propres objectifs ou l'épanouissement de sa propre personnalité n'est pas une maxime qui puisse devenir universelle. La raison en est simple: nous utilisons tous les jours les autres personnes, et donc leur corps humain, comme moyens pour atteindre nos fins ou celles des autres. Par exemple, on utilise le corps de son épouse comme moyen pour avoir ses propres enfants ${ }^{38}$. En travaillant pour le compte d'autrui, notre corps est utilisé comme moyen pour les propres fins de cet autrui.

On peut transformer la phrase de Kant en loi universelle en introduisant un concept pivot de la théorie des obligations: celui du consentement. Réécrivons la maxime de Kant de la façon suivante:

Agis de telle sorte que tu traites l'humanité, soit dans ta propre personne soit dans celle des autres, toujours comme une fin et jamais comme un moyen sans leur consentement.

Elle rappelle la position de Nozick qui écrit:
Les contraintes secondaires de l'action reflètent le principe kantien sous-jacent selon lequel les individus sont des fins et non pas seulement des moyens: ils ne peuvent pas être sacrifiés ou utilisés pour atteindre les fins d'une autre personne sans leur consentement. Les individus sont inviolables ${ }^{39}$.

La règle du consentement rend cette maxime universelle. Du fait même que la personnalité est incorporée dans un corps humain et est donc inséparable de ce corps humain, l'analogie entre la personnalité comme fin de l'individu et qui concourt à son épanouissement et son corps humain comme moyen pour atteindre ses fins et donc l'épanouissement de sa personnalité nous permet de justifier un droit de propriété sur soi à l'égal d'un droit de propriété sur un objet.

Cette maxime de Kant, corrigée par le principe du consentement, affirme explicitement que la décision de l'usage de son corps humain comme moyen pour réaliser ses propres fins ou celles des autres est dans les mains de la personne elle-même. On reconnait implicitement, cette fois, un droit de propriété de la personne sur elle-même. C'est la personne qui décide de ses propres fins, elle décide aussi des moyens pour les atteindre, en conséquence elle décide de la façon dont elle va utiliser son propre corps humain. Ce consentement est opposable à tous les tiers pris individuellement ou en groupe (dont l'État) lorsqu'ils veulent utiliser le corps humain d'une personne pour atteindre leurs propres fins ${ }^{40}$.

Cette approche est en phase avec le point de vue de l'économiste pour qui les choses et les êtres humains sont substituables comme le démontre le comportement des individus. La dichotomie entre personne et objet lui est totalement incompréhensible pour une raison simple: les choses et les personnes sont des substituts ou des moyens pour atteindre les fins que chacun poursuit ${ }^{41}$.

Il est important de comprendre la chose suivante: les individus ne désirent pas les objets pour eux-mêmes, mais pour les services qu'ils rendent. Quand nous utilisons un lit, ce n'est pas le lit, en tant qu'objet, que nous consommons $^{42}$ mais le service qu'il rend: se reposer. En revanche, le service rendu par le lit de Marie-Antoinette est tout autre: il est symbolique. On admire le lit sur lequel se reposait la femme de Louis XVI, on ne s'y allonge pas.

36. E. Kant, Fondements de la métaphysique des mours [1785], V. Delbos (trad.), Paris, Delagrave, 1965, deuxième section, p. 136.

37. Une telle interdiction n'a guère de sens, puisque la sélection des gènes se fait en se mariant. La résistance des parents aux mariages interraciaux ou interclasses sociales associées à l'avortement d'embryons présentant des tares génétiques montrent bien que l'on peut modifier génétiquement sa descendance. La pratique des organismes génétiquement modifiés (OGM) chez les plantes peut tout à fait être étendue à l'espèce humaine et elle le sera un jour si la survie de l'espèce est en jeu.

38. Il ne faut pas désespérer du progrès biotechnologique, il arrivera peut-être un jour où les enfants seront produits dans des couveuses; on passe déjà à la mère porteuse et le chemin - comme pour la machine à laver le linge sale - est déjà tracé si le coût d'opportunité du temps d'une épouse continue d'augmenter. On a déjà assisté à cela : l'épouse lave le linge, ensuite la lavandière et enfin la machine à laver le linge.

39. R. Nozick, Anarchy, State, and Utopia, Oxford, B. Blackwell, 1974, p. 31 (nous traduisons et soulignons).

40. Cela n'interdit pas de partager les fins, mais la coopération pour les atteindre est soumise au principe du consentement. Nous préférons le mot consentement à celui d'autonomie de la volonté qui renvoie au concept de rationalité individuelle. Le consentement ne nécessite pas d'être rationnel parce que la rationalité n'est pas universalisable alors que le consentement l'est.

41. Que ce soit par l'échange, le don ou l'usage de la violence pour s'emparer du corps des autres ou du fruit de leur travail. L'échange nécessite le consentement des deux parties, le don nécessite le consentement d'une seule partie, le donneur, et l'usage de la violence, individuellement ou en groupe (syndicalistes, hommes d'État, mafieux, voleurs à la sauvette, douaniers, inspecteurs des impôts, terroristes, etc.) pour s'emparer du bien d'autrui, se fait souvent sans le consentement de la victime.

42. Cela vaut aussi pour les biens alimentaires: on ne consomme pas un poisson mais les ingrédients contenus dans le poisson qui renouvellent l'énergie du corps humain. La demande de repas diététiques en est la preuve par neuf. 
De la même façon, on ne consomme pas une automobile, mais les kilomètres qu'elle permet de parcourir indépendamment des autres services qu'elle peut rendre dans le bois de Boulogne. Quand on embauche une femme de ménage, on ne la consomme pas, ce sont les services qu'elle rend que nous consommons: les services domestiques. On consomme les services rendus par les objets ou les êtres humains, non les objets ou les êtres humains eux-mêmes. Ces services sont rendus par unité de temps et sont définitivement subjectifs. Les objets ou les êtres humains sont donc substituables entre eux. Cette idée que l'on consomme les services rendus par un objet ou par une personne entraîne quatre propositions fondamentales:

- les services rendus s'expriment toujours par unité de temps, c'est-à-dire la période pendant laquelle on consomme ce service (on utilise une femme de ménage trois fois par semaine ou six fois par mois);

- les services rendus par les objets ou par les individus sont des moyens pour arriver à des fins ${ }^{43}$. Si plusieurs objets ou êtres humains peuvent rendre le même service plus ou moins bien, ils sont, d'une certaine façon, substituables;

- les services rendus sont subjectifs. L'un voit dans le lit de Marie-Antoinette une aire de repos, l'autre y voit un symbole de la royauté;

- on ne peut échanger les services rendus par les personnes ou les objets que si les droits de propriété sur les services rendus par les personnes et les choses sont clairement définis.

De nombreuses controverses, inutiles entre économistes et non-économistes, viennent de cette méconnaissance du principe de substituabilité.

La subjectivité des services rendus est aussi une caractéristique essentielle qui divise souvent, par de vaines querelles, les économistes et les non-économistes. Les services rendus par un objet, ou par un être humain, ne se définissent pas en termes physiques, mais seulement d'après l'opinion que les individus professent à leur égard. Ainsi, une médaille en or peut être un objet de décoration, un moyen de paiement, une récompense symbolique ou un objet qui sert à caler le pied d'un fauteuil bancal. Une parcelle de terrain bien exposée peut sembler être le lieu approprié à la culture de la vigne. Mais cette spécificité est une constatation qui n'est pas indépendante du lieu, du temps, des connaissances ou des anticipations qu'ont les gens sur l'utilisation alternative de ce terrain. Il peut être utilisé non seulement pour la vigne mais aussi, compte tenu de la proximité d'un lac, loué comme camping. Les services rendus par les objets ou les personnes servent à ce que les gens pensent qu'ils doivent servir ${ }^{44}$. Les tra- vaux récents de la neurologie cognitive confirment cette approche du Prix Nobel d'économie Friedrich Hayek ${ }^{45}$.

Par ailleurs, les fins poursuivies par les individus sont infiniment variées. Elles incluent des besoins alimentaires, intellectuels, sexuels et émotionnels et non pas seulement monétaires. Même si les aspects monétaires sont importants comme moyens pour atteindre les fins poursuivies. Ils peuvent être sacrifiés à d'autres buts : loisirs, méditation, contemplation, dévouement aux autres, etc. L'information complète sur toutes les alternatives auxquelles chaque individu fait face n'est pas exigée. Être constamment cohérent dans ses décisions ne l'est pas non plus. Information complète et cohérence des choix ne sont pas des prémisses de la rationalité économique mais le résultat d'un comportement qui confronte le coût d'opportunité à s'informer et/ou à être cohérent dans ses choix aux gains attendus. Il est donc parfaitement rationnel d'être ignorant ou incohérent dans ses choix ${ }^{46}$.

Les êtres humains sont des individus. C'est l'individu qui a une conscience, une identité, des besoins, des talents, une volonté. Chaque individu naît seul et meurt seul. C'est lui qui a des préférences ou des valeurs. C'est lui seul qui, de façon ultime, sait ce qui est bon pour lui. Cela ne veut pas dire qu'il ne se trompe pas ou que des amis ne puissent savoir, mieux que lui, ce qui est bon pour lui-même. Mais c'est lui qui vit une existence séparée, qui en supportera les peines et les plaisirs. C'est lui qui fait des choix et, s'il en a la liberté, qui prend des décisions: c'est l'individu qui agit. L'individu n'a pas non plus le don d'ubiquité. Lorsqu'il assiste à un cours d'économie à Paris, il ne peut assister à un cours d'anglais à New York à la même heure. Il n'est pas non plus immortel: une journée est composée de vingt-quatre heures et non de vingt-six. La rareté du temps est un fait, elle impose des choix entre différentes alternatives. Autre contrainte: les ressources nécessaires pour réaliser les fins poursuivies ne sont pas en quantités illimitées, et, malheureusement, nous ne sommes pas seuls à les convoiter. Enfin, et on ne saurait trop souligner cet aspect, c'est lui seul qui contrôle et «possède» son corps humain. Un tiers peut agir à la place de l'individu, peut essayer d'influencer la volonté de l'individu pour qu'il use de son corps dans une certaine direction soit par la menace ou par la récompense, mais de manière ultime c'est l'individu lui-même qui agit. Il est en fait «en possession" de son corps.

Si les individus partagent la plupart des mêmes fins, l'ensemble des fins poursuivies par chaque individu est unique. Du fait même de l'existence séparée de chaque individu, il est difficile à l'économiste de porter un jugement de valeur sur ces fins multiples et subjectives. Il les considère donc souvent comme des données et il les traite de manière égale. Ainsi, l'activité d'un criminel ou

43. Voir L. Robbins, Essai sur la nature et la signification de la science économique, I. Krestowski (trad.), Paris, Librairie de Médicis, 1947.

44. Voir F. Hayek, «Le caractère subjectif des données dans les sciences sociales", in Scientisme et sciences sociales: essai sur le mauvais usage de la raison, R. Barre (trad.), Paris, Plon, 1953, chap. III.

45. Voir L. Naccache, Perdons-nous connaissance? De la mythologie à la neurologie, Paris, O. Jacob, 2010.

46. Et vouloir corriger par la réglementation cette incohérence ou ignorance peut être néfaste par les effets pervers qu'elle engendre. 
d'un homme politique est analysée comme celle d'un offreur de travail ordinaire qui maximise sa satisfaction en arbitrant entre loisirs et consommation. Le drogué, le paysan spécialisé dans la production de drogue et le dealer ou les trafiquants d'organes destinés à la transplantation, les passeurs de frontières ou les hommes d'Église, passeurs d'éternité, sont considérés de la même manière que de simples consommateurs, producteurs ou intermédiaires. En cela, l'économiste est méthodologiquement amoral ou plus exactement aborde la compréhension de l'économie ou du droit, sciences morales par excellence, sans a priori moral ${ }^{47}$.

\section{Conflit sur l'usage du corps humain, droit du premier occupant et droit de propriété sur soi}

Revenons à l'idée de Descartes. Le corps humain est une machine biologique dans laquelle la personnalité est incorporée ${ }^{48}$. La personne qui habite dans cette matière vivante est aussi propriétaire des ressources qui y sont incorporées. Si elles sont valorisées par le marché, alors les exploiter rend un service à l'offreur comme au demandeur car si tous deux consentent à un transfert c'est qu'ils pensent tous deux en tirer un bénéfice. Ce corps humain peut être réparé (prothèse, transplantation d'un organe, implant dentaire), modifié (chirurgie esthétique, transsexualité), comme on le ferait d'une voiture ou d'un logement. Par analogie, il est naturel de penser que le corps humain est traité, dans les faits, par celui qui l'habite comme un objet. Si l'on pousse le concept d'inviolabilité de la personne dans sa totalité, toutes ces transformations du corps de l'individu devraient être interdites. Or, ce n'est pas le cas. Il existe de nombreuses exceptions à la règle ${ }^{49}$. Il y a donc un conflit sur les usages du corps humain entre le sujet qui habite ce corps humain et les autres personnes qui veulent exploiter les ressources qu'il contient ${ }^{50}$.

Prenons un individu, Julie, dont le corps humain est convoité par plusieurs personnes: Pierre, Paul et Amandine. Chacun pense à un usage différent du corps de Julie. Amandine désire utiliser Julie pour la beauté de son corps, Paul aimerait exploiter les talents de chanteuse de Julie et Pierre veut utiliser Julie comme domestique, mère porteuse et préceptrice de ses enfants qu'elle aura mis au monde. Enfin, Julie désire consacrer l'usage de son corps à prier Dieu dans un couvent. Le conflit a lieu à la fois entre Julie et ses trois personnalités mais aussi entre Pierre, Paul et Amandine qui convoitent la même ressource: le corps humain de Julie.

L'économiste va alors, par analogie avec d'autres ressources rattachées au sol (mine d'or, de pétrole) dont la demande a raréfié l'offre, utiliser le concept de droit de propriété. Ce dernier résout le conflit sur qui décide de l'usage du sol et donc, dans notre cas, du corps humain de Julie. L'échange de ce droit de propriété par le consentement des parties permet de mettre la ressource rare demandée (un rein destiné à la transplantation, la mise au monde d'un enfant) dans les mains de la personne qui pense en faire le meilleur usage. C'est le mécanisme de marché.

L'économiste n'a pas de prévention philosophique ou religieuse à propos des choses ou des objets et des personnes. Il fait cette analogie pour expliquer les phénomènes qu'il observe. Il ne fait pas cette dichotomie entre un objet et une personne (ou sujet) pour la raison simple que les individus consomment ou produisent les services rendus par des objets et/ou des personnes. Seule façon, pour lui, d'expliquer pourquoi le lave-linge a remplacé la lavandière ou le progrès technologique en matière de robotisation va bouleverser nos habitudes de production et de consommation ${ }^{51}$.

Face à cette convoitise sur l'usage de son corps, Julie dispose de deux stratégies (dans un face-à-face avec Paul ou Pierre ou Amandine): elle résiste de manière agressive à l'égard de l'un de ces individus ou elle se soumet au désir de la personne qui lui fait face. Julie mesure 1,90 m et pèse $90 \mathrm{~kg}$ tout en muscle, Paul, Pierre ou Amandine mesurent $1,65 \mathrm{~m}$ et pèsent $65 \mathrm{~kg}$. La tentation est grande pour Julie de gifler Paul (ou Pierre ou Amandine) autant de fois qu'il le faut pour ne plus être importunée par leur proposition. C'est souvent la réaction attendue de la part d'un observateur, la force fait le droit de propriété ${ }^{52}$.

Julie dicte sa loi et décide de l'usage de son propre corps humain. Mais cette stratégie suppose d'avoir beaucoup d'informations sur la personne avec qui elle entre en relation. Paul a des cousins et chacun d'eux pèse en moyenne

47. Les économistes sont divisés sur ce point. Les ingénieurs économistes sont comme les juristes des positivistes au sens où ils veulent une méthodologie libre des jugements de valeur en oubliant que cette position épistémologique est aussi un jugement de valeur.

48. Tous les organes destinés à la transplantation, les produits renouvelables (sang, placenta, embryon, cheveux, etc.) ou le corps humain tout entier après décès peuvent être vendus sur un marché. Ils peuvent aussi être loués: sexe (prostituée), voix (chanteur), utérus (mère porteuse), bras (manouvrier) ou jambes (footballeur), cerveau (enseignant-chercheur), etc. Il est difficile de nier que le corps humain n'est pas un moyen (un instrument) pour atteindre des fins, le 10o mètres des Jeux olympiques peut être gagné par des coureurs amputés des deux jambes sous les genoux grâce à deux prothèses en carbone. C'est vrai aussi des produits dopants.

49. Certains produits du corps humain ne sont pas hors commerce, en particulier les produits renouvelables, sang excepté (en France), comme les cheveux, le placenta, les ongles, les dents, etc.

50. Les progrès de la biotechnologie, depuis une génération, ont permis des usages du corps humain inenvisageables autrefois. Ces nouveaux usages sont devenus des ressources rares convoitées par tous. Cela ne diffère pas fondamentalement des ressources incorporées dans le sol comme le pétrole, l'uranium, l'or et autres métaux précieux, etc. Ils ne prennent de la valeur que parce que l'on a découvert des usages auxquels on ne pensait pas auparavant. Cette course à l'exploitation des ressources minières a rapidement soulevé la question des droits de propriété sur le sous-sol.

51. La libération de la femme des tâches domestiques doit beaucoup au lave-linge comme au micro-ondes, la production d'enfants en couveuse libérera la femme de la gestation; la calculette fait d'un nul en calcul mental l'égal d'un champion des "Chiffres et des lettres».

52. «Le droit est la politique de la force» est un slogan bien connu des juristes positivistes (Rudolf von Jhering, François Gény, et d'une manière plus nuancée Georges Ripert). 
$100 \mathrm{~kg}$ et est champion de karaté. Ils enlèvent Julie et la séquestrent dans la maison close d'Amandine. Avant d'agir, Julie se forge des anticipations sur le comportement des autres personnes: seront-elles Faucon ou Colombe? Si les autres ont une attitude agressive et si elle joue le faucon, c'est la guerre. Si elle anticipe chez l'autre un comportement de soumission, son intérêt est d'être agressive. À l'inverse, si l'autre est agressif, elle se soumet, car après tout il peut avoir des cousins experts en krav-maga. Les deux peuvent aussi coopérer, s'ils partagent les mêmes objectifs, par exemple avoir des enfants.

Ce conflit est très dépendant de l'incertitude qui prévaut sur le comportement d'autrui. Il peut dégénérer en une guerre qui empêche de tirer les profits attendus de l'usage du corps de Julie. Cette situation ne permet pas aux uns et aux autres d'allouer au mieux les ressources tirées de leur corps humain comme moyen d'atteindre leurs fins. Comment résoudre ce conflit? Très simplement. Une règle d'appropriation parfaitement observable, non ambiguë et universalisable à toutes situations pour le présent comme pour le futur, émerge de l'interaction spontanée entre les individus: la règle du premier occupant. Cette règle du premier occupant ou du premier en possession ${ }^{53}$ est largement connue des juristes.

Une fois reconnue cette règle du premier occupant, et le droit de propriété sur soi qui en découle (ce qui est loin d'être le cas dans la réalité du positivisme contemporain), le mécanisme de marché ou de l'échange volontaire peut se mettre en route.

Partons d'une situation où Julie est propriétaire de son corps. Peu entreprenante, elle ne tire pas un profit maximum de l'exploitation de son corps. En revanche, Paul, qui est un redoutable découvreur de talent, a trouvé une utilisation de la voix mélodieuse de Julie (les cordes vocales sont des parties du corps humain qui ne sont pas hors commerce, toujours deux poids deux mesures), qui rapporte beaucoup plus de revenus et d'épanouissement dans la chanson par rapport à l'alternative choisie: prier Dieu dans un couvent. Il peut alors proposer à Julie de lui louer sa voix ou de l'acheter avec un droit d'exclusivité à $v \mathrm{e}^{54}$. Il y met un prix élevé car il espère en tirer de hauts revenus via les concerts et les produits dérivés.

Si elle y consent, Paul achète à Julie l'usage de sa voix à un prix supérieur au profit matériel et spirituel obtenu en cloîtrant son corps humain dans un couvent, mais inférieur aux revenus que Paul pense en tirer. Mais Paul n'est pas seul. Amandine et Pierre peuvent aussi proposer à Julie une autre utilisation de son corps (pour Pierre avoir des enfants avec Julie et pour Amandine disposer de sa beauté pour mettre en valeur sa maison close). La propriété du corps de Julie est dans ses mains et non celles de quelqu'un d'autre. Il faut donc obtenir son consentement pour déplacer «l'usage» de son corps de la prière dans un couvent à la chanson populaire ou à la prostitution. En conséquence, les compétiteurs, Pierre et Amandine, doivent offrir un prix qui non seulement doit convaincre Julie de vendre une partie de son corps à l'un d'entre eux, mais qui dissuade les autres, ici Paul, de proposer un prix plus élevé 55 . Ce prix couvre nécessairement les risques pris par chacun d'eux dans l'exploitation de tout ou partie du corps de Julie. Un principe d'arbitrage est à l'œuvre: il y a une opportunité de profit à saisir en déplaçant les droits de propriété ou une fraction de ces droits dans les mains de Paul (Pierre ou Amandine) pour exploiter, parmi toutes les utilisations possibles de son corps, celle qui donne le profit maximum.

La règle du premier occupant est généralisable à tous les conflits d'appropriation entre les individus à propos de l'usage des choses et/ou des personnes. Elle donne naissance au concept de droits de propriété individuels. Ces droits sont naturels au sens où ils sont le résultat non intentionnel d'une interaction entre les hommes. Ils ne sont pas le produit d'une activité consciente des individus composant la société. Ils ne sont pas une norme extérieure aux comportements des individus. Ces droits sont des instruments de coordination des actions individuelles pour résoudre les conflits qui apparaissent lorsque plusieurs individus veulent faire un usage différent d'une ressource qu'ils convoitent. La reconnaissance d'une asymétrie non ambiguë entre les adversaires fait passer de l'état de nature hobbesien - état de guerre de tous contre tous - à un état de nature lockéen, état pacifique, où les droits de propriété fondent l'ordre social. La clé de l'émergence de la règle du premier occupant comme moyen d'attribuer les droits de propriété réside dans son observabilité, sa non-ambiguïté et son caractère universel.

\section{Cohérence du droit, inaliénabilité du libre arbitre et possession naturelle de soi}

Peut-on aliéner sa personnalité à quelqu'un d'autre ${ }^{56}$ ? L'esclavage peut-il être librement consenti? Le consentement ne suffit pas toujours pour transférer une partie ou la totalité des droits de propriété sur soi. Un droit inaliénable est un droit qui ne peut pas justement être transféré même

53. Il existe d'autres règles: la loi du plus fort (ambiguë), la loi du premier qui a découvert un usage profitable de la ressource (pas facilement observable), la priorité accordée aux femmes par bienséance (non universalisable aux relations entre femmes entre elles ou aux hommes entre eux). 54. Affaire de Johnny Halliday avec sa première maison de disques.

55. Ne croyez pas que cela n'existe pas, le prix de la mariée est un échange entre les parents du marié et les beaux-parents pour les dédommager des investissements qu'ils ont fait dans leur fille. En effet, ce sont les parents et le marié qui vont profiter des revenus générés par les sacrifices qu'ont fait les beaux-parents en investissant dans les talents de leur fille. Ce type de contrat est fréquent chez les footballeurs et les sommes atteintes sont faramineuses.

56. On peut penser au contrat d'exclusivité de Maupassant avec l'éditeur Ollendorff. Ce dernier est pratiquement "propriétaire» du corps humain de Maupassant avec les avantages - quand il écrit - et les inconvénients - quand Maupassant est soigné dans un hôpital psychiatrique aux frais de l'éditeur. Ne critiquez pas ce contrat d'exclusivité, c'est le même type de contrat qui vous lie à la clientèle électorale dont les représentants ont pris le pouvoir ou à votre époux avant les années 1965 
avec le consentement de celui qui le détient. Quelles sont les raisons d'une telle inaliénabilité bien illustrée d'une certaine manière par les droits de la personne?

Par définition un droit de propriété sur soi interdit aux autres pris individuellement ou en groupe (époux/se, syndicat et / ou État-nation) de violer ce droit. Si donc on doit respecter les droits de propriété des autres sur eux-mêmes, on ne peut aliéner le droit de propriété sur soi-même pour violer les droits d'une autre personne.

$\mathrm{Si}$ Jules cède son temps de travail, son corps et son libre arbitre à Pierre en acceptant de lui obéir en tout point, alors refuser d'obéir aux ordres de Pierre reviendrait à violer les droits de Pierre. Jules aurait donc tort de désobéir. Maintenant, si Pierre oblige Jules à violer les droits d'un tiers en lui ordonnant par exemple de tuer son épouse, une espèce de divorce à l'italienne des années $1960^{57}$, Pierre commandite un homicide ${ }^{58}$.

Si on fait respecter un tel contrat, deux conséquences morales s'ensuivent:

- si on fait respecter le premier contrat, le tueur n'est pas responsable de la violation des droits du tiers qu'il menace: c'est le commanditaire qui est responsable;

- mais le tueur, en obéissant à Pierre, viole les droits du tiers, ceux de son épouse et en refusant d'obéir, c'est-à-dire en ne respectant pas le contrat, il viole les droits du commanditaire.

Peut-on se décharger de sa responsabilité de violer les droits de propriété d'autrui en la transférant sur un autre? Le droit transféré ne doit pas conduire à des situations contradictoires. Il faut donc choisir. Ou bien certains droits dans le contrat ne sont pas aliénables (violer les droits d'un tiers au contrat), ou, si on fait respecter le contrat entre le commanditaire et l'exécutant, le tiers en question n'a aucun moyen de faire respecter ses droits de propriété sur lui-même si le contrat entre le commanditaire et l'exécutant est passé.

Toute théorie des droits de propriété sur son corps humain protège par définition les individus de la violation de leurs droits par autrui. Quel que soit le contrat passé entre un commanditaire (employeur) et l'exécutant (un employé), l'exécutant ne peut que retenir le droit de ne pas violer les droits d'autrui. Le contrat entre le commanditaire et l'exécutant est frappé de nullité. Cette règle s'impose aussi aujourd'hui à tout fonctionnaire recevant un tel ordre. Les points 10 et 11 du panier de droits de la propriété de soi sont justifiés non pas pour leurs conséquences mais par l'universalité et la compatibilité du droit.
Dans cet ordre d'idée, peut-on transférer totalement son corps à une autre personne et ce de son vivant en contrepartie d'une rémunération au bénéfice d'un tiers, époux, enfants ou voisins ${ }^{59}$ ? Supposons un tel transfert partiel ou total du corps humain de Julie. Cette dernière cède le contrôle de son corps à Amandine. Nous avons un contrat d'esclavage ou de servitude ${ }^{60}$ entre ces deux personnes. Un tel contrat n'est pas valide. Pourquoi?

Premièrement, Julie peut, par consentement, transférer le contrôle sur son corps à quelqu'un d'autre comme dans un contrat de travail. Cependant, en obéissant aux ordres de celui à qui elle a transféré ce droit, Julie ne peut pas se transformer en machine. Amandine est donc contrainte de compter sur Julie pour contrôler ce qui lui appartient: le corps humain de Julie. En fait, le transfert du corps humain n'est pas physiquement réalisé, car Julie, qui contracte avec Amandine, contrôle toujours l'objet transféré: son corps humain.

Deuxièmement, un tel contrat d'échange nécessite de transférer totalement le contrôle de soi-même à autrui. Dans un contrat d'esclavage, l'une des parties au contrat disparaît en tant que personne. Ou bien la ressource est externe et le droit de contrôler celle-ci ne pose aucun problème, ou bien la ressource que l'on cherche à contrôler est interne à un être humain, auquel cas il s'agit d'un droit d'utiliser cette ressource et non de la transférer en totalité. Votre corps humain peut être transféré pour partie (contrat de servitude), mais vous ne pouvez pas aliéner votre contrôle (ou votre libre arbitre) sur votre corps humain sans cesser d'être un humain (i.e. mort ou inconscient). Vous ne pouvez pas vous débarrasser de votre propre volonté. L'individu qui se soumet à la volonté d'un maître n'est pas encore un esclave puisque sa soumission est consentie; en revanche, s'il change d'avis plus tard, en refusant par exemple de se plier aux desiderata de son maître et que ce dernier impose par la force, à son esclave, de faire ce qu'il lui dit de faire, l'esclavage n'est plus volontaire.

La question fondamentale n'est pas de savoir si Amandine, notre tenancière de maison close, peut utiliser la force pour contraindre Julie à faire ce qu'elle lui ordonne de faire, puisque Julie y a normalement consenti, mais de savoir si ce consentement engage Julie dans n'importe quel usage de son corps. Imaginez qu'après avoir promis à Amandine de satisfaire toutes les lubies de ses clients, Julie refuse de le faire. Julie rompt sa promesse. Amandine peut-elle contraindre Julie à tenir sa promesse? Quand Amandine use de la contrainte pour obliger Julie à faire ce qu'elle avait promis de faire, est-ce que Julie peut résister

57. Divorce à l'italienne est un film italien de Pietro Germi sorti en 1961 avec Marcello Mastroianni en vedette principale.

58. Les hommes d'État eux sont coutumiers du crime commandité. Tous les jours ils agissent ainsi par l'intermédiaire de leurs fonctionnaires avec l'appui de la force publique (impôt, conscription ou meurtre de personnalités gênantes réalisé par des services secrets).

59. La vente ne peut se faire au bénéfice de l'esclave lui-même puisqu'il a vendu son corps, la somme revient dans la poche du propriétaire. En général, l'esclavage résulte d'une violence initiale et l'esclave ou le serf rachète sa liberté au bandit qui s'est approprié son corps ou prend la fuite et se réfugie dans les sauvetés ou les villes franches.

60. Pour certains les transactions faramineuses opérées par les clubs de football de joueurs ayant bénéficié des investissements en capital humain incorporés dans leurs corps humains ressemblent beaucoup à des contrats de servitude. Le contrat de mariage, au moment du divorce, en est aussi une illustration: les ex-maris doivent payer des prestations compensatoires à leurs ex-femmes. 
de plein droit? Le contrat d'échange volontaire, selon lequel Amandine peut utiliser légitimement la force pour contraindre Julie, oblige Julie à ne pas résister à Amandine. Cependant, quel que soit le contrat, dans les faits, Julie contrôle toujours son corps. Ce contrôle est toujours dans les mains de Julie et non dans celles d'Amandine, l'objet de l'échange, le contrôle du corps de Julie, n'a pas été transféré ${ }^{61}$. Il y a une contradiction et une incohérence dont on se sort justement en refusant de considérer comme valide un contrat d'esclavage volontaire. Le point $6 \mathrm{du}$ panier de droits de la propriété de la personne sur son propre corps humain bute contre l'impossibilité de transférer le contrôle du corps humain par la personne qui l'occupe à quelqu'un d'autre ${ }^{62}$.

\section{Une doctrine qui confond droit et morale ${ }^{63}$}

Tous, à un moment ou à un autre, nous faisons face au problème de savoir ce que nous «devrions» faire. En général, tuer son épouse sous le prétexte futile qu'elle a un amant n'est guère acceptable. En revanche, on accepte la pratique d'une interruption «volontaire» de grossesse (volontaire pour qui ?) si le fotus devient une gêne pour sa mère. Autant le premier jugement de valeur semble partagé par tous dans notre culture ${ }^{64}$, autant le second ne l'est pas.

Quand on affirme "vous ne devriez pas faire l'action A» ou plus brièvement «ne fait pas $\mathrm{A}$ », on porte un jugement de valeur «bien» ou «mal» sur l'action d'un individu. Quand les lois sur la bioéthique affirment:

Aucun paiement, quelle qu'en soit la forme, ne peut être alloué à celui qui se prête au prélèvement d'éléments de son corps ou à la collecte de ses produits ${ }^{65}$.

Elles signifient que celui qui exige un paiement pour prélever les éléments de son corps agit «mal» et entreprend une action «immorale». Il est souvent facile de repérer ces jugements quand les mots «bien», «mal», "moral», «immoral» sont explicitement utilisés. Mais d'autres mots sont souvent évoqués pour affirmer la même chose sans qu'on reconnaisse immédiatement un jugement de valeur identique. Les mots: «irresponsable», «immérité», «anti- social» sont des qualificatifs qui indiquent que l'individu entreprend des actions jugées comme "mauvaises».

Mais comment peut-on justifier une théorie morale tant que l'on ne sait pas ce qu'est un jugement moral, c'est-à-dire tant que l'on ne sait pas ce que veulent dire les mots «bien» ou «mal»?

D’abord, il faut vérifier si les mots «bien» ou «mal» ont une signification. Rien n'est moins sûr. Ils ne sont utilisables que pour des comportements individuels et conscients. Le gouvernement d'un pays n'agit pas, on ne peut donc évoquer les mots «bien» ou «mal» relativement à son action. Ce sont les membres de ce gouvernement qui agissent, non le gouvernement lui-même. Ainsi, on peut dire que le ministre de la Justice a mal agi et que les députés qui ont voté «oui » à sa loi ont, eux aussi, mal agi. Si la plupart des choses (un typhon, une éruption volcanique) ou des organismes vivants (un lion, un serpent) n'ont pas de conscience, on ne peut attribuer à leurs actions un jugement de valeur mais un jugement de fait. Il fut un temps, pas si éloigné, où l'on jugeait les animaux devant un tribunal pour des actions qui entraînaient de mauvaises conséquences pour les uns ou les autres.

\section{A. Droit positif ou droit moraliste?}

Pour un scientifique, les jugements de valeur «bien» ou «mal» n'ont aucun contenu cognitif. Ils ont un contenu existentiel ou émotionnel mais seraient sans valeur «scientifique». C'est le positivisme. Les juristes sont habitués à cette interprétation. En effet, leur positivisme ${ }^{66}$ a été développé par Hans Kelsen ${ }^{67}$ qui voulait effacer du droit toute idéologie politique, morale et religieuse. Il proposa une théorie limitée à l'analyse du droit positif comme un système de normes issu d'un processus politique de décisions collectives ${ }^{68}$. Si le droit est positif, cela ne veut pas dire que la morale est exclue du droit. Bien au contraire, elle entre en force par le jeu démocratique. Chaque groupe de pression et chaque faction politique cherchent à capturer le processus législatif pour imposer aux autres «sa » morale. De ce seul fait, le droit est devenu moraliste, voire immoral, en fonction de ceux qui transitoirement s'emparent du pouvoir d'écrire la loi. Les lois sur la bioéthique sont exemplaires de cette dérive. Le droit n'a pas pour objet d'imposer

61. Voir R. Barnet, The Structure of Liberty: Justice and the Rule of Law, Oxford, Clarendon Press - Oxford University Press, 1998, p. 78-79; G. H. Smith, "Inalienable Rights», Liberty Magazine, vol. 10, n 6, juillet 1997, p. 52, cité par R. Barnet, The Structure of Liberty..., p. 79.

62. Une telle proposition revient à contester le contrat de travail qui n'est pas un contrat de louage de service. Pour certains penseurs, il s'agit d'un contrat de servitude qui devrait être nul de plein droit. On comprend bien pourquoi les patrons offrent de tels contrats: il s'agit de fixer la main d'œuvre en réduisant sa mobilité liée à d'autres opportunités de vies. Cette mobilité coûte cher aux patrons ou aux actionnaires des entreprises concernées. La face bénéfique du syndicalisme (ou légitime) se rapporte au contrôle qu'exerce le patron sur chaque salarié pour faire un usage de son corps humain qui détruirait le libre arbitre et la possession naturelle de soi. La face nuisible du syndicat réside dans une entente entre salariés pour maintenir un salaire ou un prix du travail au-dessus du prix qui aurait été observé en l'absence de violence légale ou illégale.

63. Cette section prend appui sur B. Lemennicier, La morale face à l'économie, chap. I.

64. Ce n'est pas le cas dans la culture musulmane où les femmes adultères sont lapidées en place publique jusqu'à ce que mort s'ensuive.

65. Art. L. 1211-4 du Code de la santé publique.

66. Positivisme qu'il faudrait associer au mot «neutralité» à l'égard des jugements de valeur.

67. H. Kelsen, Théorie pure du droit: introduction à la science du droit, H. Thévenaz (trad.), Neuchâtel, Éditions de la Baconnière, 1953.

68. Ce processus politique de décision collective (la démocratie politique) ne filtre pas les jugements de valeur pour les écarter, bien au contraire, il les renforce en les sanctifiant par la loi. C'est bien le problème des lois sur la bioéthique. 
une morale mais de rendre compatible la poursuite par chacun de sa propre morale pour gouverner ses actions.

\section{B. La variété des approches morales}

Si les jugements de valeur «bien» ou «mal» ont une signification cognitive, il peut être intéressant de voir comment on juge du bien et du mal. Existe-t-il des théories morales suffisamment générales mais différentes qui permettent de guider nos décisions à tout moment? Il existe trois grandes façons de classer la plupart des jugements de valeur: la première est le conséquentialisme, la deuxième la déontologie et la dernière la téléologie. Elles nous permettent de détecter quelle est la morale qui sous-tend fondamentalement les lois sur la bioéthique. Ces lois sont-elles fondées sur leurs conséquences bonnes ou mauvaises (justification de la légalisation de l'avortement ou de la drogue)? Sont-elles fondées sur le respect d'une règle de déontologie exprimée par exemple par le commandement impératif: «Tu considéreras toute personne comme une fin en soi et non comme un moyen "? Sont-elles fondées sur une règle téléologique selon laquelle une fin est poursuivie pour elle-même et non comme instrument pour atteindre une autre fin, l'amitié pour l'amitié, le don pour le don?

Une théorie conséquentialiste de la morale soutient que les jugements moraux sont fondés sur les effets produits par une action. Une action est jugée morale ou immorale sur la base des conséquences qu'elle entraîne. Mais nos actions peuvent-elles être jugées morales ou immorales sur la base de leurs conséquences? C'est la première question que l'on est en droit de se poser à propos d'une vision conséquentialiste de la morale. La deuxième question soulevée par cette approche consiste à savoir sur quel critère on juge que les conséquences sont bonnes ou mauvaises: l'efficacité, l'équité, le juste? Il faut aussi spécifier pour qui les résultats des actions sont bons ou mauvais. S'agit-il du bonheur de l'individu lui-même? S'agit-il du bonheur d'un groupe d'individus: les plus pauvres, les prolétaires, les gens noirs de peau ou les femmes? Ou s'agit-il plus simplement du bonheur du plus grand nombre?
Une théorie déontologique nie ce qu'affirme le conséquentialisme. Une action individuelle est bonne ou mauvaise indépendamment de ses conséquences. Les pacifistes affirment haut et fort que toute utilisation des armes à feu est mauvaise en soi, même si la conséquence a pour résultat le décès du pacifiste en question faute de pouvoir se défendre contre une agression. Au lieu de juger du bien et du mal en termes de conséquences, on en juge en termes de respect de certaines règles d'action. La vision déontologique de la morale est purement procédurale. Elle a l'avantage de ne pas porter de jugement de valeur, ni sur les fins, ni sur les règles puisque les règles elles-mêmes résultent de cette métarègle. Elle laisse aux parties en présence le soin de définir leurs propres morales ou leurs propres systèmes d'évaluation des conséquences de leurs actions. C'est le point de vue que nous avons adopté dans ce texte lorsque nous avons discuté du concept de personne. En revanche, quand nous avons évoqué l'aspect nuisible de la doctrine sous-jacente aux lois sur la bioéthique, nous avons adopté un point de vue conséquentialiste sous la forme d'un impératif hypothétique.

Il existe cependant une autre vision, celle dite téléologique, qui s'oppose aux deux précédentes. Ni conséquentialiste, ni déontologique, elle affirme qu' «est bien » tout acte qui est poursuivi pour lui-même, qui est sa propre finalité. Ainsi, est «bien» le comportement d'amitié s'il est poursuivi par un individu pour la chaleur des relations qu'il procure avec son supérieur hiérarchique et non pour la promotion qu'il en attend. «Est mal» tout comportement d'amitié qui serait instrumental vis-à-vis d'autres finalités. Il en va de même avec la beauté, l'intégrité, le courage, la connaissance, le don, etc. En ce sens-là, ce comportement par définition n'est pas conséquentialiste, il en est l'antonyme. Il ne s'agit pas non plus d'une règle, d'un devoir, il n'y a aucune raison à chaque fois que l'on rencontre un supérieur hiérarchique de lui offrir son amitié désintéressée.

Les lois sur la bioéthique reposent donc, finalement, sur cette morale téléologique ${ }^{69}$. Le don doit être désintéressé, l'amour de l'autre doit être oblatif. On cultive le don pour le don et non pas pour les bénéfices monétaires ou non monétaires (valorisation du surmoi, réciprocité et troc d'appariement) que l'on en attend.

69. Une morale téléologique prend ses racines dans la tradition de l'éthique d'Aristote. La poursuite du bien se fait en conformité avec la nature propre de l'être humain, c'est-à-dire d'un être rationnel, égoïste et libre de ses choix. On peut renvoyer à la philosophe Ayn Rand en citant les extraits suivants de sa vision morale de la vie d'un homme en tant qu'homme: «Puisque la raison est l'instrument fondamental de la survie de l'homme en tant qu'homme, ce qui est propre à la vie d'un être humain rationnel est bon, ce qui est contraire est mauvais [...]. La rationalité est la vertu principale de l'homme avec les trois valeurs fondamentales: la raison, les objectifs, l'estime de soi [...]. L'homme - chaque homme - est une fin en soi, et non un moyen pour les fins des autres. Il doit vivre pour lui-même, sans se sacrifier pour autrui, ni sacrifier autrui à ses propres fins [...]. Il doit travailler pour son intérêt personnel avec la poursuite de son propre bonheur comme principe moral le plus élevé de sa vie» (A. Rand, The Virtue of Selfishness, New York, New American Library, 1961, p. 13-35; nous traduisons). On peut aussi inclure dans cette vision l'existentialisme de Jean-Paul Sartre. Ce dernier écrit : «Car nous voulons dire que l'homme existe d'abord, c'est-à-dire que l'homme est d'abord ce qui se jette vers un avenir, et ce qui est conscient de se projeter dans l'avenir. L'homme est d'abord un projet qui se vit subjectivement» (J.-P. Sartre, L'existentialisme est un humanisme [1946], Paris, Nagel, 1970, p. 23). L'être humain est donc orienté vers un but (le don pour le don), mais sa condition est telle qu'il réalise ce projet en relation avec les autres et la question de la compatibilité de son projet avec celui des autres (chacun réalise ses anticipations) est posée. Cette question a sa réponse dans la déontologie. Par exemple, celle de la morale de Kant combinée avec celle de Nozick (que nous utilisons) prescrit (impératif catégorique) une règle de conduite de la part des individus: ne jamais utiliser les autres comme moyen pour atteindre ses propres fins sans leur consentement (règle prescrite par les lois sur la bioéthique mais qui ne rend pas compatibles les projets des individus faute de l'instauration d'un mécanisme de marché pour assurer la compatibilité des plans entre offreurs et demandeurs). 
Si donc l'on accorde un sens cognitif aux jugements de valeur, cela veut dire que certains jugements de valeur sont «vrais» alors que d'autres sont «faux». On peut alors rechercher quels sont les principes généraux qui permettent de trancher sur une base rationnelle entre une approche conséquentialiste, déontologique et téléologique? L'un de ces principes est l'universalité. La morale qui guide les actions des individus doit être telle qu'elle peut se transformer en loi universelle dans l'espace et le temps. L'autre principe suppose que la morale suivie par chaque individu n'est pas relative à l'individu lui-même. Elle est objective ou absolutiste.

En quoi la morale du don pour le don est-elle une morale universelle et «objectivement vraie» pour que l'on puisse l'imposer de façon autoritaire à tous par l'intermédiaire du législateur? Est-ce que la morale du don pour le don existe indépendamment des individus ${ }^{70}$ ? La réponse est claire et négative. Elle n'est pas universelle ni universalisable au sens où si tout le monde pratiquait cette morale, personne n'aurait rien à donner. La survie d'une société repose sur la nature égoïste de l'être humain et non sur l'altruisme oblatif. De la poursuite d'un mal (l'égoïsme) ${ }^{71}$ naît un bienfait pour le reste de la société. C'est l'argument conséquentialiste d'Adam Smith illustré par la célèbre phrase:

Ce n'est pas de la bienveillance du boucher, du marchand de bière et du boulanger, que nous attendons notre dîner, mais bien du soin qu'ils apportent à leurs intérêts. Nous ne nous adressons pas à leur humanité, mais à leur égoïsme; et ce n'est jamais de nos besoins que nous leur parlons, $c^{\prime}$ 'est toujours de leur avantage ${ }^{72}$.

La morale téléologique est aussi relative à chaque individu. En effet, elle ne peut rejeter comme immoral un comportement individuel dont l'objet serait la poursuite du mal pour le mal au sens où aucun intérêt financier n'interfère avec la conduite de l'individu.

Mais alors si la vérité éthique de la téléologie est relative à l'individu, elle l'est aussi à la société, à la culture ou à l'espèce humaine du moment. Le relativisme s'oppose à l'absolutisme ou à l'objectivisme. Les jugements moraux ne sont pas universels ni indépendants des individus. Les valeurs morales sont privées, subjectives et l'individu est la seule mesure de toutes choses. Cependant, si l'individu est à la base de la vérité des jugements moraux, personne ne peut se tromper ou avoir des jugements de valeur "faux». Comment alors comparer et trancher entre différents jugements moraux? Une telle implication est radicale. La morale devient purement arbitraire, subjective et individualiste.

Si une action est morale seulement parce qu'une autorité P (le Comité consultatif national d'éthique, l'électeur médian dans une démocratie) dit qu'elle est morale, elle est subjective et relative aux membres de ce comité. Elle n'a donc aucune valeur «objective» ni absolutiste.

Le paradoxe réside dans le fait que ces trois façons d'approcher la morale sont à la fois antinomiques et complémentaires. Toute morale qui juge du bien ou du mal à propos d'un comportement ne peut passer outre aux bonnes ou aux mauvaises conséquences des actes individuels sur eux-mêmes et sur les autres, aussi bien la déontologie que la téléologie. Réciproquement, peut-on justifier des actes qui violent des règles déontologiques sur les bonnes conséquences attendues pour l'individu lui-même ou pour les autres? Doit-on sacrifier la vie de centaines de personnes pour en sauver dix mille? Peut-on absoudre moralement les comportements d'un tueur en série au prétexte que ses actes n'étaient pas assortis d'un intérêt monétaire ou non monétaire?

Nous n'avons pas à rentrer dans ces conflits ni à imposer par la loi une morale quelconque. Il ne s'agit pas en effet de savoir s'il existe un code moral «objectif»" qui pourrait être découvert et sur lequel tout le monde pourrait se mettre d'accord, ni de savoir si chacun peut suivre la morale qu'il croit «vraie» compte tenu de ses propres critères d'évaluation, il s'agit de faire en sorte que chacun puisse poursuivre sa vision de la morale sans empêcher quelqu'un d'autre de suivre la sienne. C'est la position de Henry Hazlitt ${ }^{73}$.

Si l'on admet l'existence séparée des individus et le libre arbitre, si l'on constate que les fins des individus sont profondément subjectives et que les morales qui sous-tendent les actions des individus le sont tout autant, la question fondamentale de la méta-éthique n'est pas de savoir si l'une est supérieure à l'autre ou s'il existe une morale objective qui s'impose à tous, mais de trouver une procédure ou une règle qui rendent compatibles entre elles la diversité des morales qui guident les actions des individus. De ce processus par lequel les différentes morales vont devenir compatibles entre elles émerge

La question de juger des conséquences des règles sur la compatibilité des projets est entière. La morale conséquentialiste peut devenir une métarègle instrumentale pour décider quelles règles déontologiques sont bonnes ou «efficaces » pour atteindre l'objectif considéré: atteindre un ordre social où chacun réalise ses anticipations c'est-à-dire ses projets de vie. Les trois approches morales peuvent être utilisées fonctionnellement d'une manière complémentaire. L'économie est exemplaire sur ce point. S'il s'agit de la science des contrats, l'économie est déontologique. S'il s'agit de la science des choix, elle est téléologique en insistant sur la rationalité et l'égoïsme comme vertus. Mais, paradoxalement, elle est aussi conséquentialiste au niveau des métarègles puisque les deux morales précédentes sont souvent justifiées par leurs «bonnes» conséquences en permettant d'atteindre un ordre social où chacun peut réaliser ses anticipations.

70. En revanche, la déontologie répond à ce critère. Le Décalogue, le code de Hammurabi, les lois sacrées de Manu en témoignent. Connaissez-vous une société où rompre ses promesses, mentir, tricher, voler, user de la violence, tuer sont les règles? Certains répondront par l'affirmative puisque ces règles s'appliquent largement dans les sociétés politiques ou politisées. Mais habituellement les sociétés civiles (et civilisées) reposent sur le respect des promesses, l'honnêteté, la vérité et la paix.

71. Pour d'autres, il s'agit au contraire d'une vertu: A. Rand, The Virtue of Selfishness.

72. A. Smith, Richesse des nations [1776], J.-G. Courcelle-Seneuil (éd.), Paris, Guillaumin et C ${ }^{\mathrm{ie}}, 1888$, p. 22.

73. H. Hazlitt, The Foundations of Morality, New York, D. Van Nostrand Company Inc., 1964, p. 356, $\$ 11$. 
une certaine vision de ce qu'il est bien ou mal de faire dans une société ouverte à toutes les formes de moralité individuelle.

Où est donc la solution? Vous l'avez déjà devinée. En effet, si les questions de morale sont in fine une discussion conflictuelle sur l'usage de votre corps humain (y compris les idées) ou sur celui d'autrui, il s'agit d'un conflit d'appropriation. Il suffit alors d'établir un droit de propriété sur ce corps humain et de l'attribuer au premier occupant.

Le respect du droit de propriété sur soi est le moyen par lequel on rend compatible la diversité des morales. De la cohérence et de l'universalité de ce droit de propriété sur soi émerge une éthique «objective» qui guide les actions individuelles.

Or, si on adopte cette façon de porter un jugement sur les diverses morales, celle avancée par le Comité consultatif national d'éthique et indirectement par le législateur en matière de science de la vie est incohérente, car le concept de personne sur laquelle elle est fondée présente trop de contradictions; elle est non universalisable parce que le concept de personnalité n'est pas neutre et dépend de la façon dont les personnes elles-mêmes se définissent comme personne et enfin elle est contredite par les actes spontanés des individus qui s'approprient de plus en plus leur corps humain et désirent disposer librement de ses éléments ou de ses produits.

On peut contraster cette morale avec l'idée simple que chacun est propriétaire de soi-même. Cette notion de droit, qui consiste en une appropriation de son corps, est cohérente. Le corps humain est un objet comme un autre dont le propriétaire est parfaitement identifié. Elle est universalisable, tout être humain potentiel ou non, tout esprit incorporé dans une machine biologique ou non bénéficie d'un droit de propriété sur cette machine parce qu'il en est l'occupant ou qu'il en a la possession. Elle n'est pas contredite par les actes spontanés des individus puisque ce droit de propriété accompagne la privatisation de plus en plus grande de l'individu sur ses actes comme sur l'usage de son corps. Cette reconnaissance d'un droit de propriété sur soi légitime la libre disposition de son corps et de ses éléments. Elle ne confond pas morale et droit.

Attribuer la propriété du corps humain à celui qui y habite ne dit rien des fins que poursuit l'individu et ne porte pas de jugement sur les valeurs qui vont guider les actions de l'individu qui pilote ce corps humain. Ce n'est pas non plus socialement nuisible bien au contraire. Cela permet de ne pas hypothéquer l'avenir de l'espèce humaine et protège mieux les libertés individuelles que le concept de personne, cher aux philosophes de notre droit contemporain. Enfin, ce n'est pas philosophiquement mal fondé. Le droit de propriété sur soi est facilement définissable. Il est souple et peut être décomposé. On peut louer son corps. On peut en vendre certains éléments ou la totalité après décès. On peut le détruire, le transformer, etc. Le droit de propriété privée sur soi-même est un droit non une morale. Chacun peut utiliser la morale qu'il veut pour guider ses actions. Mais il ne pourra le faire qu'en respectant le droit de propriété d'autrui sur son propre corps. En ce sens, le droit de propriété privée sur soi rend compatibles les différents points de vue moraux. C’est sa supériorité. Il est fondé sur la tolérance.

On peut conclure cet article par un rappel fondamental. Ce n'est pas au droit ni à la loi de trancher ou d'imposer une morale. Pour trancher entre ces diverses morales, c'est-à-dire pour savoir laquelle est bonne ou mauvaise, il faut une procédure, et des règles permettant d'écarter certaines d'entre elles. L'éthique est l'instrument intellectuel qui permet d'une façon rationnelle de trancher entre des points de vue moraux différents. On exige d'une morale, soutenue par un groupe de pression quelconque (les scientifiques, les religieux, les moralistes, les juristes, les économistes), qu'elle soit au moins cohérente, universalisable et d'une certaine manière non contredite par les actes spontanés des individus. 